Journal of Mathematical Physics, Analysis, Geometry

2014, vol. 10, No. 4, pp. 451-484

\title{
On the Fluctuations of Entries of Matrices whose Randomness is due to Classical Groups
}

\author{
V. Vasilchuk \\ B. Verkin Institute for Low Temperature Physics and Engineering \\ National Academy of Sciences of Ukraine \\ 47 Lenin Ave., Kharkiv, 61103, Ukraine \\ E-mail: vasilchuk@ilt.kharkov.ua
}

Received December 20, 2013, revised September 9, 2014

\begin{abstract}
We consider first the $n \times n$ random matrices $H_{n}=A_{n}+U_{n}^{*} B_{n} U_{n}$, where $A_{n}$ and $B_{n}$ are Hermitian, having the limiting normalized counting measure (NCM) of eigenvalues as $n \rightarrow \infty$, and $U_{n}$ is unitary uniformly distributed over $U(n)$. We find the leading term of asymptotic expansion for the covariance of elements of resolvent of $H_{n}$ and establish the Central Limit Theorem for the elements of sufficiently smooth test functions of the corresponding linear statistics. We consider then analogous problems for the matrices $W_{n}=S_{n} U_{n}^{*} T_{n} U_{n}$, where $U_{n}$ is as above and $S_{n}$ and $T_{n}$ are nonrandom unitary matrices having limiting NCM's as $n \rightarrow \infty$.

Key words: Random matrices, Central Limit Theorem, Limit Laws.

Mathematics Subject Classification 2010: 60F05, 15B52 (primary); 15A18 (secondary).
\end{abstract}

\section{Introduction}

In the recent decades the asymptotic properties of eigenvalues of various random matrices have been of great interest (see, e.g., [3]). This paper deals with the entries of functions of two classes of random matrices.

The first class consists of the $n \times n$ Hermitian random matrices

$$
H=A+U^{*} B U
$$

and

$$
\tilde{H}=V^{*} A V+U^{*} B U .
$$

Note that here and below we do not write the subindex $n$ in various matrices.

(C) V. Vasilchuk, 2014 
We assume that $A$ and $B$ are non-random Hermitian matrices, $V$ and $U$ are independent unitary random matrices uniformly distributed over the unitary group $U(n)$, i.e., having the normalized Haar measure as their probability law. Besides, we assume without lost of generality that $A$ and $B$ are diagonal:

$$
A_{j k}=\alpha_{j} \delta_{j k}, B_{j k}=\beta_{j} \delta_{j k}
$$

Note that while the probability distributions of eigenvalues of (1.1) and (1.2) coincide, this is not the case, in general, for entries.

The second class consists of the matrices

$$
W=S U^{*} T U
$$

and

$$
\hat{W}=V^{*} S V U^{*} T U
$$

Here we assume that $S$ and $T$ are unitary non-random and diagonal, $V$ and $U$ are the same as in (1.2).

Let

$$
G(z)=\left(A+U^{*} B U-z I\right)^{-1}
$$

be the resolvent of (1.1). We use the same notation for the resolvents of (1.1) and (1.2) and for the resolvents of the ensembles (1.3) and (1.4). The complex variable $z$ will vary over the set $\operatorname{Im} z \neq 0$ for (1.1) and (1.2) and over $|z| \neq 1$ for (1.3) and (1.4).

Introduce the normalized eigenvalue counting measure (NCM) of any $n \times n$ Hermitian matrix $M$ as follows:

$$
N_{n}(\Omega)=\#\left\{\lambda_{l}^{(n, M)} \in \Omega, l=1, \ldots, n\right\} n^{-1},
$$

where $\Omega \subset \mathbb{R}$ and $\left\{\lambda_{l}^{(n, M)}\right\}$ are the eigenvalues of $M$. Analogously, for any $n \times n$ unitary matrix $Q$, the NCM is defined for any interval $\Omega \subset \mathbb{T}=\{z \in \mathbb{C}:|z|=1\}$.

A standard tool to study the fluctuations of eigenvalues are the linear eigenvalue statistics $[2-4]$

$$
\mathcal{N}_{n}[\varphi]:=\operatorname{Tr} \varphi\left(H_{n}\right)=\sum_{l=1}^{n} \varphi\left(\lambda_{l}^{H_{n}}\right)=n \int_{-\infty}^{+\infty} \varphi(\lambda) N_{n}(d \lambda)
$$

where $\varphi: \mathbb{R} \rightarrow \mathbb{C}$ is a measurable and bounded function, e.g.,

$$
\mathcal{N}_{n}\left[\varphi_{z}\right]=\operatorname{Tr} G(z), \varphi_{z}(\lambda)=(\lambda-z)^{-1} .
$$

In $[3,4]$, this study is based on the following two useful propositions: 
Proposition 1.1. If $\varphi: \mathbb{R} \rightarrow \mathbb{R}, \varphi \in \mathcal{L}$-vector space with the norm $\|\cdot\|$ and we have:

- the variance $V_{n}[\varphi]=\operatorname{Var}\left\{\mathcal{N}_{n}[\varphi]\right\}$ admits uniform in the $n$ bound $V_{n}[\varphi] \leq$ $C\|\varphi\|^{2}$ for all $\varphi \in \mathcal{L}$

- there exists a dense linear manifold $\mathcal{L}_{1} \subset \mathcal{L}$ such that the Central Limit Theorem is valid for $\mathcal{N}_{n}^{\circ}[\varphi]$ with $\varphi \in \mathcal{L}_{1}$, i.e., if $Z_{n}[x \varphi]=\mathbf{E}\left\{e^{i x \mathcal{N}_{n}^{\circ}[\varphi]}\right\}$, then there exists a continuous quadratic functional $V: \mathcal{L}_{1} \rightarrow \mathbb{R}_{+}$such that uniformly in $x$ on any compact

$$
\lim _{n \rightarrow \infty} Z_{n}[x \varphi]=e^{-x^{2} V[\varphi] / 2}, \forall \varphi \in \mathcal{L}_{1},
$$

then $V$ admits a continuous extension to $\mathcal{L}$ and the Central Limit Theorem is valid for all $\mathcal{N}_{n}^{\circ}[\varphi]$ with $\varphi \in \mathcal{L}$.

Proposition 1.2. For any $s>0$ and

$$
\|\varphi\|_{s}^{2}=\int_{-\infty}^{+\infty}(1+2|k|)^{2 s}|\hat{\varphi}(k)|^{2} d k, \hat{\varphi}(k)=\frac{1}{2 \pi} \int_{-\infty}^{+\infty} e^{i k x} \varphi(x) d x
$$

we have $\operatorname{Var}\left\{\mathcal{N}_{n}[\varphi]\right\} \leq$

$$
\leq C\|\varphi\|_{s} \int_{0}^{+\infty} d y e^{-y} y^{2 s-1} \int_{-\infty}^{+\infty} \operatorname{Var}\{\operatorname{Tr} G(x+i y)\} d x .
$$

These tools allow one to prove the Central Limit Theorem for sufficiently wide classes of test functions of the linear eigenvalue statistics, provided that it is proved for some special classes, e.g., analytic or even $\varphi_{z}$ of (1.7), for a certain domain of $z$ 's. Accordingly, we will first prove the Central Limit Theorem for the elements $G_{j j}$ of the resolvent and then generalize it by using the analogs of the above propositions. Note that $G_{j j}$ and $\mathcal{N}_{n}[\varphi]_{j j}:=\varphi(H)_{j j}\left(\right.$ or $\left.\varphi(W)_{j j}\right)$ are not the linear eigenvalue statistics (1.6) but just certain statistics of eigenvalues and eigenvectors. We will thus essentially use the linearity of $\mathcal{N}_{n}[\varphi]_{j j}$ in $\varphi$.

\section{Covariance of Resolvents of (1.1) and (1.2)}

We will need the Stieltjes transform

$$
g(z)=n^{-1} \operatorname{Tr} G=\int_{-\infty}^{+\infty} \frac{N_{n}(d \lambda)}{\lambda-z}
$$

of the NCM of (1.1). 
Let $N_{n, A}$ and $N_{n, B}$ be the Normalized Counting Measures of $A$ and $B$. We assume that the measures converge weakly to the probability measures $N_{A}$ and $N_{B}$ :

$$
\lim _{n \rightarrow \infty} N_{n, A}=N_{A}, \lim _{n \rightarrow \infty} N_{n, B}=N_{B} .
$$

We will also need the following result obtained in $[1,2]$ :

Theorem 2.1. Consider the $n \times n$ random Hermitian matrices (1.1) (or (1.2)), where $A$ and $B$ are non-random, satisfying condition (2.1). Then there exists a non-random measure $N$ such that the Normalized Counting Measures of eigenvalues of (1.1) (or (1.2)) converge weakly with probability 1 to $N$.

Moreover, the Stieltjes transform

$$
f(z)=\int_{-\infty}^{+\infty} \frac{N(d \lambda)}{\lambda-z}, \operatorname{Im} z \neq 0,
$$

of $N$ is a unique solution of the system

$$
\left\{\begin{array}{l}
f(z)=f_{A}\left(h_{B}(z)\right) \\
f(z)=f_{B}\left(h_{A}(z)\right) \\
f^{-1}(z)=z-h_{A}(z)-h_{B}(z),
\end{array}\right.
$$

where

$$
f_{A, B}(z)=\int_{-\infty}^{+\infty} \frac{N_{A, B}(d \lambda)}{\lambda-z},
$$

$f$ is the Stieltjes transform of a probability measure and $h_{A, B}$ are analytic in $\mathbb{C} \backslash \mathbb{R}$ functions verifying the conditions

$$
f(z)=-z^{-1}+o\left(z^{-1}\right), h_{A, B}(z)=z+o(z), z \rightarrow \infty .
$$

The first result of this paper is as follows:

Theorem 2.2. Consider the random matrices (1.1) and (1.2), where $A$ and $B$ are non-random, satisfying condition (2.1), and $\|A\|,\|B\| \leq M<\infty$. Then we have for $G_{k k}$ and $z_{1,2} \in \mathbb{C} \backslash \mathbb{R}$ that

$$
\operatorname{Cov}\left\{G_{k k}\left(z_{1}\right), G_{k k}\left(z_{2}\right)\right\}=\frac{1}{n} S_{n}\left(z_{1}, z_{2}\right)+r_{n}\left(z_{1}, z_{2}\right),
$$

where in the case of (1.1),

$$
S_{n}\left(z_{1}, z_{2}\right)=\frac{\left(\left(G_{A}\left(h_{B}\left(z_{1}\right)\right)-G_{A}\left(h_{B}\left(z_{2}\right)\right)\right)_{k k}\right)^{2}}{\delta f}\left(\frac{1}{\delta z}-\frac{1}{\delta h_{B}}\right),
$$


and in the case of (1.2),

$$
S_{n}\left(z_{1}, z_{2}\right)=\frac{\delta f}{\delta z}-f\left(z_{1}\right) f\left(z_{2}\right),
$$

in which

$$
\begin{gathered}
\delta z=z_{1}-z_{2}, \delta f=f\left(z_{1}\right)-f\left(z_{2}\right), \\
\delta h_{B}=h_{B}\left(z_{1}\right)-h_{B}\left(z_{2}\right), G_{A}(z)=(A-z I)^{-1},
\end{gathered}
$$

and the remainder $r_{n}\left(z_{1}, z_{2}\right)$ admits the bound

$$
\left|r_{n}\left(z_{1}, z_{2}\right)\right| \leq C / n^{3 / 2}
$$

where $C$ is independent of $n$ and is finite if $\min \left\{\left|\operatorname{Im} z_{1}\right|,\left|\operatorname{Im} z_{2}\right|\right\}>0$.

$\mathrm{R}$ e $\mathrm{m}$ a $\mathrm{r} \mathrm{k}$ 2.3. In the case $A=\alpha I,(1.1)$ and (1.2) coincide, and hence (2.6) and (2.7) coincide as well. Indeed, we have

$$
G_{A}\left(h_{B}(z)\right)=\frac{1}{\alpha-h_{B}(z)} I=f_{A}\left(h_{B}(z)\right) I=f(z) I
$$

and, by the resolvent identity, we have

$$
\frac{G_{A}\left(h_{B}\left(z_{1}\right)\right)-G_{A}\left(h_{B}\left(z_{2}\right)\right)}{\delta h_{B}}=G_{A}\left(h_{B}\left(z_{1}\right)\right) G_{A}\left(h_{B}\left(z_{2}\right)\right)=f\left(z_{1}\right) f\left(z_{2}\right) I .
$$

Moreover, if also $B=\beta I$, then the r.h.s. in (2.6) is zero since

$$
h_{B}(z)=z-\beta, \delta h_{B}=\delta z
$$

i.e., there is no randomness at all. And this is only the case where the leading terms (2.6) and (2.7) of the covariances are both zero.

$\mathrm{R}$ e $\mathrm{m}$ a $\mathrm{r} \mathrm{k}$ 2.4. The results similar to Theorem 2.2 for various random matrix ensembles with independent matrix entries were recently obtained in [7-9]. In these papers, as well as in Theorem 2.2, the asymptotic regime for the variance is a classical one, i.e., $n^{-1}$, but the validness of the Central Limit Theorem (CLT) strongly depends on the probability distributions of the matrix entries. In Gaussian case, CLT is valid, otherwise it is not valid in general.

E x a m p l e 2.5. Consider (1.2) with $A=B$ and $N_{A}(d \lambda)=\alpha \delta(\lambda+1)+$ $(1-\alpha) \delta(\lambda-1), 0 \leq \alpha \leq 1$. Then (2.2) reduces to the quadratic equation

$$
\left(z^{2}-4\right) f^{2}(z)-4(2 \alpha-1) f(z)-1=0
$$


with the solution

$$
f(z)=\frac{2(2 \alpha-1)}{z^{2}-4}-\frac{\sqrt{4(2 \alpha-1)^{2}+z^{2}-4}}{z^{2}-4}
$$

and the resulting measure

$N(d \lambda)=\left[\alpha-\frac{1}{2}\right]_{+} \delta(\lambda+2)+\left[\frac{1}{2}-\alpha\right]_{+} \delta(\lambda-2)+\frac{\sqrt{(\lambda-\lambda)\left(\lambda_{+}-\lambda\right)}}{\pi(\lambda+2)(2-\lambda)} \chi_{\left[\lambda_{-}, \lambda_{+}\right]}(\lambda) d \lambda$,

where $\chi_{\left[\lambda_{-}, \lambda_{+}\right]}$is the indicator of the segment $\left[\lambda_{-}, \lambda_{+}\right]$, and

$$
\lambda_{ \pm}= \pm 2 \sqrt{1-(2 \alpha-1)^{2}}, \quad[x]_{+}= \begin{cases}x, & x>0 \\ 0, & x \leq 0\end{cases}
$$

Thus, we have in this case:

$$
\begin{aligned}
S\left(z_{1}, z_{2}\right)= & \frac{z_{1}+z_{2}}{u\left(z_{1}\right) u\left(z_{2}\right)}\left(-2(2 \alpha-1)+\frac{\left.1+\frac{4(2 \alpha-1)^{2}\left(u\left(z_{1}\right)+u\left(z_{2}\right)\right)}{u\left(z_{1}\right) u\left(z_{2}\right)}\right)}{\frac{w\left(z_{1}\right)}{u\left(z_{1}\right)}+\frac{w\left(z_{2}\right)}{u\left(z_{2}\right)}}\right) \\
& -\frac{\left(2(2 \alpha-1)-w\left(z_{1}\right)\right)\left(2(2 \alpha-1)-w\left(z_{2}\right)\right)}{u\left(z_{1}\right) u\left(z_{2}\right)}
\end{aligned}
$$

where $u(z)=z^{2}-4, w(z)=\sqrt{4(2 \alpha-1)^{2}+z^{2}-4}$.

\section{3. $\quad$ Proof of Theorem 2.2}

In what follows, by $\langle\ldots\rangle$ we denote the integral in $U$ (conditional expectation) with respect to the normalized Haar measure of $U(n)$ and by $\mathbf{E}\{\ldots\}$, the double integral over $U$ and $V$ (expectation) in (1.2). We denote by $a^{\circ}=a-\mathbf{E}\{a\}$ the centered random variable. We will use the following two facts [1-3].

Proposition 3.1. Let $\mathcal{H}_{n}$ be the space of the $n \times n$ Hermitian matrices and $\Phi: \mathcal{H}_{n} \rightarrow \mathbb{C}$ be a continuously differentiable function. Then we have for any $X \in \mathcal{H}_{n}$ :

$$
\left\langle\Phi^{\prime}\left(U^{*} M U\right) \cdot\left[X, U^{*} M U\right]\right\rangle=0,
$$

where

$$
\left[M_{1}, M_{2}\right]=M_{1} M_{2}-M_{1} M_{2} .
$$

Proposition 3.2. Let $\Phi: U(n) \rightarrow \mathbb{C}$ be a continuously differentiable function. Then

$$
\operatorname{Var}\left\{\Phi\left(U_{n}\right)\right\}:=\mathbf{E}\left\{\left|\Phi\left(U_{n}\right)\right|^{2}\right\}-\left|\mathbf{E}\left\{\Phi\left(U_{n}\right)\right\}\right|^{2} \leq \frac{1}{n} \sum_{j, k=1}^{n} \mathbf{E}\left\{\left|\Phi^{\prime}\left(U_{n}\right) \cdot E^{(j, k)} U_{n}\right|^{2}\right\}
$$


where $\left\{E^{(j, k)}\right\}_{j, k=1}^{n}$ is a canonical basis in the space $\mathcal{M}_{n}$ of all $n \times n$ matrices: $E^{(j, k)}=\left\{E_{p q}^{(j, k)}\right\}_{p, q=1}^{n}, E_{p q}^{(j, k)}=\delta_{j p} \delta_{k q}$.

P r o o f of Theorem 2.2. We start from the case (1.1). In Proposition 3.1, choosing $\Phi=\left(\operatorname{Tr} E^{(k, k)} G\left(z_{1}\right)\right)^{\circ} G_{a c}\left(z_{2}\right)$ and using the resolvent identity for the pair $(H, A)$, we obtain

$$
\begin{aligned}
& \left\langle\left(\operatorname{Tr} E^{(k, k)} G\left(z_{1}\right)\right)^{\circ}\left(G\left(z_{2}\right)\left[X, U^{*} B U\right] G\left(z_{2}\right)\right)_{a c}\right\rangle \\
+ & \left\langle\left(\operatorname{Tr} E^{(k, k)} G\left(z_{1}\right)\left[X, U^{*} B U\right] G\left(z_{1}\right)\right) G_{a c}\left(z_{2}\right)\right\rangle=0 .
\end{aligned}
$$

Then we take $X=E^{(a, b)}$ and apply the operation $n^{-1} \sum_{a=1}^{n}$ to the result. This yields the matrix equality

$$
\begin{gathered}
\left\langle G_{k k}^{\circ}\left(z_{1}\right) \delta_{n, B}\left(z_{2}\right) G\left(z_{2}\right)\right\rangle=\left\langle G_{k k}^{\circ}\left(z_{1}\right) g_{n}\left(z_{2}\right) U^{*} B U G\left(z_{2}\right)\right\rangle \\
+n^{-1}\left\langle\left[U^{*} B U, G\left(z_{1}\right) E^{(k, k)} G\left(z_{1}\right)\right] G\left(z_{2}\right)\right\rangle,
\end{gathered}
$$

where

$$
\delta_{n, B}=n^{-1} \operatorname{Tr} U^{*} B U G .
$$

Rewrite $U^{*} B U G$ by using the resolvent identity for the pair $(H, A)$ as $U^{*} B U G=$ $I-(A-z) G$. After regrouping the terms, we get

$$
\begin{aligned}
f_{n}\left(z_{2}\right)(A & \left.-h_{n, B}\left(z_{2}\right) I\right)\left\langle G_{k k}^{\circ}\left(z_{1}\right) G\left(z_{2}\right)\right\rangle=\frac{1}{n}\left\langle\left[U^{*} B U, G\left(z_{1}\right) E^{(k, k)} G\left(z_{1}\right)\right] G\left(z_{2}\right)\right\rangle \\
+ & \left\langle G_{k k}^{\circ}\left(z_{1}\right) g_{n}^{\circ}\left(z_{2}\right) U^{*} B U G\left(z_{2}\right)\right\rangle-\left\langle G_{k k}^{\circ}\left(z_{1}\right) \delta_{n, B}^{\circ}\left(z_{2}\right) G\left(z_{2}\right)\right\rangle,
\end{aligned}
$$

where

$$
f_{n}(z)=\mathbf{E}\left\{g_{n}(z)\right\}, \quad h_{n, B}(z)=z-\frac{\mathbf{E}\left\{\delta_{n, B}(z)\right\}}{f_{n}(z)} .
$$

In view of $\|A\|,\|B\| \leq M$, we have the bounds

$$
\begin{aligned}
|| G(z) \| & \leq \frac{1}{|\operatorname{Im} z|},\left|g_{n}(z)\right| \leq \frac{1}{|\operatorname{Im} z|}, f_{n}(z)=-\frac{1}{z}+o\left(\frac{1}{z}\right), \\
\left|\delta_{n, B}(z)\right| & \leq \frac{m_{B}^{(1)}}{|\operatorname{Im} z|}, m_{A, B}^{(k)}=\sup _{n} \int_{-\infty}^{+\infty}|\lambda|^{k} N_{n, A, B}(d \lambda) \leq M^{k}, \\
\left|g_{n}(z)\right| & \geq|z|^{-1}\left(1-\frac{2 M}{|\operatorname{Im} z|}\right),\left|h_{n, B}(z)\right| \geq|z|\left(1-\frac{M}{|\operatorname{Im} z|-2 M}\right)
\end{aligned}
$$

valid uniformly in $n$ in the domain

$$
\begin{aligned}
\Gamma_{\alpha, \beta} & =\{z \in \mathbb{C}:|\operatorname{Re} z| \leq \alpha|\operatorname{Im} z|,|\operatorname{Im} z| \geq \beta\}, \beta>(2 \alpha+6) \max \left\{M, M^{2}\right\} \\
\text { and } k & =0, \ldots, 4
\end{aligned}
$$


Thus,

$$
\left|\operatorname{Im} h_{n, B}(z)\right| \geq \beta \frac{\beta-(\alpha+3) M}{\beta-2 M},
$$

and the matrix $A-h_{B}(z) I$ in (3.2) has the inverse

$$
\widetilde{G}_{A}(z)=\left(A-h_{n, B}(z) I\right)^{-1}=G_{A}\left(h_{n, B}(z)\right)
$$

bounded uniformly in $n$ and $z \in \Gamma_{\alpha, \beta}$ :

$$
\left\|\widetilde{G}_{A}(z)\right\| \leq\left(\beta \frac{\beta-(\alpha+3) M}{\beta-2 M}\right)^{-1}
$$

Furthermore, multiplying (3.2) by $\widetilde{G}_{A}\left(z_{2}\right)$ from the left then applying the operation $\operatorname{Tr} E^{(k, k)}$. and regrouping the terms, we obtain the relation

$$
\operatorname{Cov}\left\{G_{k k}\left(z_{1}\right), G_{k k}\left(z_{2}\right)\right\}=\left\langle G_{k k}^{\circ}\left(z_{1}\right) G_{k k}\left(z_{2}\right)\right\rangle=\frac{1}{n} \gamma\left(z_{1}, z_{2}\right)+R_{1},
$$

where

$$
\begin{aligned}
\gamma\left(z_{1}, z_{2}\right)= & \frac{1}{f_{n}\left(z_{2}\right)}\left(\left\langle\widetilde{G}_{A}\left(z_{2}\right) U^{*} B U G\left(z_{1}\right)\right\rangle_{k k}\left\langle G\left(z_{1}\right) G\left(z_{2}\right)\right\rangle_{k k}\right. \\
& \left.-\left\langle\widetilde{G}_{A}\left(z_{2}\right) G\left(z_{1}\right)\right\rangle_{k k}\left\langle G\left(z_{1}\right) U^{*} B U G\left(z_{2}\right)\right\rangle_{k k}\right), \\
R_{1}= & \frac{1}{f_{n}\left(z_{2}\right)}\left(\mathbf{E}\left\{G_{k k}^{\circ}\left(z_{1}\right) g_{n}^{\circ}\left(z_{2}\right) \widetilde{G}_{A}\left(z_{2}\right) U^{*} B U G\left(z_{2}\right)\right\}\right. \\
& -\mathbf{E}\left\{G_{k k}^{\circ}\left(z_{1}\right) \delta_{n, B}^{\circ}\left(z_{2}\right) \widetilde{G}_{A}\left(z_{2}\right) G\left(z_{2}\right)\right\} \\
& +n^{-1} \mathbf{C o v}\left\{\left(\widetilde{G}_{A}\left(z_{2}\right) U^{*} B U G\left(z_{1}\right)\right)_{k k},\left(G\left(z_{1}\right) G\left(z_{2}\right)\right)_{k k}\right\} \\
& \left.-n^{-1} \mathbf{C o v}\left\{\left(\widetilde{G}_{A}\left(z_{2}\right) G\left(z_{1}\right)\right)_{k k},\left(G\left(z_{1}\right) U^{*} B U G\left(z_{2}\right)\right)_{k k}\right\}\right) .
\end{aligned}
$$

Now the Schwarz inequality for the expectation $\mathbf{E}\{\ldots\}$ and (3.8) yields for $z$, $z_{1,2} \in \Gamma_{\alpha, \beta}$,

$\left|R_{1}\right| \leq$

$$
\begin{aligned}
& |z| \operatorname{Var}^{1 / 2}\left\{G_{k k}(z)\right\}\left(M \operatorname{Var}^{1 / 2}\left\{g_{n}(z)\right\}+\operatorname{Var}^{1 / 2}\left\{\delta_{n, B}(z)\right\}\right)\left(\beta^{2} \frac{\beta-(\alpha+3) M}{\beta-2 M}\right)^{-1} \\
& +\frac{1}{n} \frac{|z|}{1-\frac{2 M}{\beta}}\left(\operatorname{Var}^{1 / 2}\left\{\left(\widetilde{G}_{A}\left(z_{2}\right) U^{*} B U G\left(z_{1}\right)\right)_{k k}\right\} \operatorname{Var}^{1 / 2}\left\{\left(G\left(z_{1}\right) G\left(z_{2}\right)\right)_{k k}\right\}\right. \\
& \left.+\operatorname{Var}^{1 / 2}\left\{\left(\widetilde{G}_{A}\left(z_{2}\right) G\left(z_{1}\right)\right)_{k k}\right\} \operatorname{Var}^{1 / 2}\left\{\left(G\left(z_{1}\right) U^{*} B U G\left(z_{2}\right)\right)_{k k}\right\}\right)
\end{aligned}
$$


Besides, by Proposition 3.2, we have

$$
\begin{aligned}
\operatorname{Var}\left\{G_{k k}(z)\right\} & \leq \frac{1}{n} \sum_{j, t=1}^{n} \mathbf{E}\left\{\left|\operatorname{Tr} E^{(k, k)} G U^{*}\left(E^{(t, j)} B-B E^{(j, t)}\right) U G\right|^{2}\right\} \\
& =\frac{1}{n} \sum_{j, t=1}^{n} \mathbf{E}\left\{\left|\left(B U G E^{(k, k)} G U^{*}\right)_{j t}-\left(U G E^{(k, k)} G U^{*} B\right)_{t j}\right|^{2}\right\} \\
& \leq \frac{4 M^{2}}{n \beta^{4}}
\end{aligned}
$$

and, analogously,

$$
\begin{aligned}
\operatorname{Var}\left\{\left(\widetilde{G}_{A}\left(z_{2}\right) U^{*} B U G\left(z_{1}\right)\right)_{k k}\right\} & \leq \frac{8 M^{4}}{n \beta^{6}}\left(\frac{\beta-(\alpha+3) M}{\beta-2 M}\right)^{-2}, \\
\operatorname{Var}\left\{\left(G\left(z_{1}\right) G\left(z_{2}\right)\right)_{k k}\right\} & \leq \frac{8 M^{2}}{n \beta^{6}} \\
\operatorname{Var}\left\{\left(\widetilde{G}_{A}\left(z_{2}\right) G\left(z_{1}\right)\right)_{k k}\right\} & \leq \frac{4 M^{2}}{n \beta^{6}}\left(\frac{\beta-(\alpha+3) M}{\beta-2 M}\right)^{-2} \\
\operatorname{Var}\left\{\left(G\left(z_{1}\right) U^{*} B U G\left(z_{2}\right)\right)_{k k}\right\} & \leq \frac{16 M^{4}}{n \beta^{6}} .
\end{aligned}
$$

It was shown in $[1,2]$ that

$$
\operatorname{Var}\left\{g_{n}(z)\right\}, \operatorname{Var}\left\{\delta_{n, B}(z)\right\}=O\left(n^{-2}\right) .
$$

Thus, we have that

$$
R_{1}=O\left(n^{-3 / 2}\right)
$$

uniformly in $z \in \Gamma_{\alpha, \beta}$.

It was shown in $[1,2]$ that

$$
\langle G(z))\rangle=\widetilde{G}_{A}(z)+O\left(n^{-1}\right) I .
$$

Thus, using the resolvent identity, we can write the matrix relations:

$$
\begin{aligned}
\left\langle\widetilde{G}_{A}\left(z_{2}\right) U^{*} B U G\left(z_{1}\right)\right\rangle & =\widetilde{G}_{A}\left(z_{2}\right)\left\langle U^{*} B U G\left(z_{1}\right)\right\rangle \\
& =\frac{z_{1}-h_{n, B}\left(z_{1}\right)}{\delta h_{n, B}}\left(\widetilde{G}_{A}\left(z_{1}\right)-\widetilde{G}_{A}\left(z_{2}\right)\right)+O\left(n^{-1}\right) I, \quad(3.17) \\
\left\langle G\left(z_{1}\right) G\left(z_{2}\right)\right\rangle & =\frac{1}{\delta z}\left(\widetilde{G}_{A}\left(z_{1}\right)-\widetilde{G}_{A}\left(z_{2}\right)\right)+O\left(n^{-1}\right) I, \\
\left\langle\widetilde{G}_{A}\left(z_{2}\right) G\left(z_{1}\right)\right\rangle & =\widetilde{G}_{A}\left(z_{2}\right)\left\langle G\left(z_{1}\right)\right\rangle=\frac{\left(\widetilde{G}_{A}\left(z_{1}\right)-\widetilde{G}_{A}\left(z_{2}\right)\right)}{\delta h_{n, B}}+O\left(n^{-1}\right) I, \\
\left\langle G\left(z_{1}\right) U^{*} B U G\left(z_{2}\right)\right\rangle & =\frac{\left(z_{1} \widetilde{G}_{A}\left(z_{1}\right)-z_{2} \widetilde{G}_{A}\left(z_{2}\right)\right)}{\delta z}-\left\langle G\left(z_{1}\right) A G\left(z_{2}\right)\right\rangle+O\left(n^{-1}\right) I .
\end{aligned}
$$


It was shown in [2] that

$$
\left\langle G\left(z_{1}\right) A G\left(z_{2}\right)\right\rangle=A \widetilde{G}_{A}\left(z_{1}\right) \widetilde{G}_{A}\left(z_{2}\right)-\frac{\delta\left(\frac{\Delta_{B}}{f}\right) \delta \Delta_{A}}{\delta z \delta f} \widetilde{G}_{A}\left(z_{1}\right) \widetilde{G}_{A}\left(z_{2}\right)+O\left(n^{-1}\right) I,
$$

where

$$
\begin{aligned}
& \Delta_{B}=\mathbf{E}\left\{\delta_{n, B}\right\}, \Delta_{A}=\mathbf{E}\left\{n^{-1} \operatorname{Tr} A G\right\}, \delta \Delta_{A, B}=\Delta_{A, B}\left(z_{1}\right)-\Delta_{A, B}\left(z_{2}\right), \\
& \delta\left(\frac{\Delta_{B}}{f}\right)=\frac{\Delta_{B}\left(z_{1}\right)}{f\left(z_{1}\right)}-\frac{\Delta_{B}\left(z_{2}\right)}{f\left(z_{2}\right)} .
\end{aligned}
$$

Using this relation, we obtain

$$
\left\langle G\left(z_{1}\right) U^{*} B U G\left(z_{2}\right)\right\rangle=\frac{\delta \Delta_{B}}{\delta z \delta f}\left(\widetilde{G}_{A}\left(z_{1}\right)-\widetilde{G}_{A}\left(z_{2}\right)\right)+O\left(n^{-1}\right) I .
$$

Substituting these expressions into (3.9), we obtain (2.5) with (2.6).

Now we pass to the case (1.2). Repeating the same steps, we arrive (cf $(3.2))$ to

$$
\begin{aligned}
f_{n}\left(z_{2}\right)( & \left.V^{*} A V-h_{n, B}\left(z_{2}\right) I\right)\left\langle G_{k k}^{\circ}\left(z_{1}\right) G\left(z_{2}\right)\right\rangle \\
& =f_{n}\left(z_{2}\right)\left\langle G_{k k}^{\circ}\left(z_{1}\right)\right\rangle \\
& +n^{-1}\left\langle\left[U^{*} B U, G\left(z_{1}\right) E^{(k, k)} G\left(z_{1}\right)\right] G\left(z_{2}\right)\right\rangle \\
& +\left\langle G_{k k}^{\circ}\left(z_{1}\right) g_{n}^{\circ}\left(z_{2}\right) U^{*} B U G\left(z_{2}\right)\right\rangle \\
& -\left\langle G_{k k}^{\circ}\left(z_{1}\right) \delta_{n, B}^{\circ}\left(z_{2}\right) G\left(z_{2}\right)\right\rangle .
\end{aligned}
$$

Then, multiplying this relation by $\widetilde{G}_{V^{*} A V}\left(z_{2}\right)=\left(V^{*} A V-h_{n, B}\left(z_{2}\right)\right)^{-1}$ from the left then applying the operation $\operatorname{Tr} E^{(k, k)}$., taking the average $\mathbf{E}\{\ldots\}$ of the result and regrouping the terms, we obtain the relation (cf. (3.9))

$$
\begin{aligned}
& \operatorname{Cov}\left\{G_{k k}\left(z_{1}\right), G_{k k}\left(z_{2}\right)\right\}=\operatorname{Cov}\left\{G_{k k}\left(z_{1}\right),\left(\widetilde{G}_{V^{*} A V}\left(z_{2}\right)\right)_{k k}\right\} \\
& +\frac{1}{n} \frac{1}{f_{n}\left(z_{2}\right)}\left(\mathbf{E}\left\{\widetilde{G}_{V^{*} A V}\left(z_{2}\right) U^{*} B U G\left(z_{1}\right)\right\}_{k k} \mathbf{E}\left\{G\left(z_{1}\right) G\left(z_{2}\right)\right\}_{k k}\right. \\
& \left.-\mathbf{E}\left\{\widetilde{G}_{V^{*} A V}\left(z_{2}\right) G\left(z_{1}\right)\right\}_{k k} \mathbf{E}\left\{G\left(z_{1}\right) U^{*} B U G\left(z_{2}\right)\right\}_{k k}\right)+R_{1},
\end{aligned}
$$

where $R_{1}$ can be estimated by (3.12), and by the same arguments (Proposition 3.2), we have again (3.16) $R_{1}=O\left(n^{-3 / 2}\right)$. On the other hand, repeating our procedure for

$$
\Phi=\left(\operatorname{Tr} E^{(k, k)} \widetilde{G}_{V^{*} A V}\left(z_{1}\right)\right)^{\circ} G_{a c}\left(z_{2}\right),
$$


we obtain

$$
\begin{aligned}
& \operatorname{Cov}\left\{G_{k k}^{\circ}\left(z_{1}\right),\left(\widetilde{G}_{V^{*} A V}\left(z_{2}\right)\right)_{k k}\right\} \\
= & \operatorname{Cov}\left\{\left(\widetilde{G}_{V^{*} A V}\left(z_{1}\right)\right)_{k k},\left(\widetilde{G}_{V^{*} A V}\left(z_{2}\right)\right)_{k k}\right\}+R_{2} \\
= & \operatorname{Cov}\left\{\left(V^{*} \widetilde{G}_{A}\left(z_{1}\right) V\right)_{k k},\left(V^{*} \widetilde{G}_{A}\left(z_{2}\right) V\right)_{k k}\right\}+R_{2},
\end{aligned}
$$

where

$$
\begin{gathered}
R_{2}=\frac{1}{f_{n}\left(z_{2}\right)}\left(\mathbf{E}\left\{\left(\widetilde{G}_{V^{*} A V}\left(z_{1}\right)\right)_{k k}^{\circ} g_{n}^{\circ}\left(z_{2}\right) \widetilde{G}_{A}\left(z_{2}\right) U^{*} B U G\left(z_{2}\right)\right\}\right. \\
-\mathbf{E}\left\{\left(\widetilde{G}_{V^{*} A V}\left(z_{1}\right)\right)_{k k}^{\circ} \delta_{n, B}^{\circ}\left(z_{2}\right) \widetilde{G}_{A}\left(z_{2}\right) G\left(z_{2}\right)\right\} .
\end{gathered}
$$

Analogously, we obtain

$$
R_{2}=O\left(n^{-3 / 2}\right)
$$

Besides, it was shown in [3] that

$$
\begin{aligned}
\int_{V \in U(n)} \bar{V}_{l_{1} k} V_{l_{2} k} d V & =n^{-1} \delta_{l_{1} l_{2}}, \\
\int_{V \in U(n)} \bar{V}_{l_{1} k} V_{l_{2} k} \bar{V}_{l_{3} k} V_{l_{4} k} d V & =(n(n+1))^{-1}\left(\delta_{l_{1} l_{2}} \delta_{l_{3} l_{4}}+\delta_{l_{1} l_{4}} \delta_{l_{2} l_{3}}\right) .
\end{aligned}
$$

Thus, we have

$$
\begin{aligned}
\mathbf{E}\left\{\left(\widetilde{G}_{V^{*} A V}(z)\right)_{k k}\right\} & =\mathbf{E}\left\{\left(V^{*} \widetilde{G}_{A}(z) V\right)_{k k}\right\} \\
& =\frac{1}{n} \operatorname{Tr} \widetilde{G}_{A}(z)=f(z)
\end{aligned}
$$

and

$$
\begin{aligned}
& \operatorname{Cov}\left\{\left(V^{*} \widetilde{G}_{A}\left(z_{1}\right) V\right)_{k k},\left(V^{*} \widetilde{G}_{A}\left(z_{2}\right) V\right)_{k k}\right\} \\
= & \frac{1}{n+1}\left(\frac{1}{n} \operatorname{Tr} \widetilde{G}_{A}\left(z_{1}\right) \widetilde{G}_{A}\left(z_{2}\right)-\frac{1}{n} \operatorname{Tr} \widetilde{G}_{A}\left(z_{1}\right) \frac{1}{n} \operatorname{Tr} \widetilde{G}_{A}\left(z_{2}\right)\right) \\
= & \frac{1}{n}\left(\frac{\delta f}{\delta h_{n, B}}-f\left(z_{1}\right) f\left(z_{2}\right)\right)+O\left(n^{-2}\right) .
\end{aligned}
$$

Moreover, taking the expectation $\mathbf{E}\{\ldots\}$ of (3.17), we obtain

$$
\begin{aligned}
\mathbf{E}\left\{\widetilde{G}_{V^{*} A V}\left(z_{2}\right) U^{*} B U G\left(z_{1}\right)\right\} & =\left(\frac{z_{1}-h_{n, B}\left(z_{1}\right)}{\delta h_{n, B}} \delta f+O\left(n^{-1}\right)\right) I, \\
\mathbf{E}\left\{G\left(z_{1}\right) G\left(z_{2}\right)\right\} & =\left(\frac{\delta f}{\delta z}+O\left(n^{-1}\right)\right) I,
\end{aligned}
$$




$$
\begin{aligned}
\mathbf{E}\left\{\widetilde{G}_{V^{*} A V}\left(z_{2}\right) G\left(z_{1}\right)\right\} & =\left(\frac{\delta f}{\delta h_{n, B}}+O\left(n^{-1}\right)\right) I, \\
\mathbf{E}\left\{G\left(z_{1}\right) U^{*} B U G\left(z_{2}\right)\right\} & =\left(\frac{\delta \Delta_{B}}{\delta z}+O\left(n^{-1}\right)\right) I .
\end{aligned}
$$

Substituting (3.20) and (3.21) into (3.18), we obtain (2.5) with (2.7).

$\mathrm{R}$ e $\mathrm{m}$ a $\mathrm{r} \mathrm{k}$ 3.3. It was proved in [5] that the functions $h_{A, B}$ in the system (2.2) are not only analytic in $\mathbb{C} \backslash \mathbb{R}$, but are also the Nevanlinna functions obeying asymptotics (2.4):

$$
h_{A, B}(z)=z+o(z), z \rightarrow \infty .
$$

Using this, we can carry out the proofs of the above theorem not only for the region $\Gamma_{\alpha, \beta}$, but for the whole $\mathbb{C} \backslash \mathbb{R}$. In this case,

$$
\left|\operatorname{Im} h_{A, B}(z)\right| \geq|\operatorname{Im} z|, z \in \mathbb{C} \backslash \mathbb{R} .
$$

Thus, once we have proved the convergence of $\left(f_{n}, h_{n, A}, h_{n, B}\right)$ to the solution of the limiting system $(2.2)$, we have $h_{n, B}(z) \rightarrow h_{B}(z), z \in \mathbb{C} \backslash \mathbb{R}$ and, hence, the matrix $\widetilde{G}_{A}$ of (3.7) admits the bound

$$
\left\|\widetilde{G}_{A}(z)\right\| \leq C
$$

uniformly in $z$ in a compact set $K \subset \mathbb{C} \backslash \mathbb{R}$ and sufficiently large $n$ with an $n$ independent $C$, finite for $\operatorname{Im} z \neq 0$. Since Proposition 3.2 provides the bounds (3.13) and (3.14) for the variances for any $z \in \mathbb{C} \backslash \mathbb{R}$, we can estimate all above remainders for any $z \in \mathbb{C} \backslash \mathbb{R}$.

\section{Central Limit Theorem for the Entries of Functions of (1.1) and (1.2)}

In this section we use (2.6) and (2.7) for the covariance to prove the Central Limit Theorem for the entries of the resolvent and then for the entries of the sufficiently smooth function of the matrices (1.1) and (1.2). The first result is

Theorem 4.1. Consider the ensembles (1.1) and (1.2) satisfying the conditions (2.1) and $\|A\|,\|B\| \leq M<\infty$, then $\sqrt{n}\left(G_{k k}(z)-\mathbf{E}\left\{G_{k k}(z)\right\}\right)$ converges in distribution to the complex Gaussian random variable $\xi(z)+i \eta(z)$ with zero mean and the covariances:

$$
\begin{aligned}
\operatorname{Var}\{\xi(z)\} & =2^{-1} \operatorname{Re}(S(z, z)+S(z, \bar{z})), \\
\operatorname{Var}\{\eta(z)\} & =-2^{-1} \operatorname{Re}(S(z, z)-S(z, \bar{z})), \\
\operatorname{Cov}\{\xi(z), \eta(z)\} & =2^{-1} \operatorname{Im}(S(z, z)-S(z, \bar{z})),
\end{aligned}
$$

where $S\left(z_{1}, z_{2}\right)$ is defined in (2.6) and (2.7) for (1.1) and (1.2), respectively. 
P r o o f. The proof follows the proof of the corresponding theorem for the fluctuations of $n g_{n}^{\circ}(z)$ in [2].

Since the proofs for the ensembles (1.1) and (1.2) coincide, let us prove the theorem only for the ensemble (1.1). Consider $\operatorname{Re} \sqrt{n} G_{k k}^{\circ}(z)$ and its characteristic function

$$
F_{n}(t)=\mathbf{E}\{\psi(t)\}, \psi(t)=\exp \left\{i t \operatorname{Re} \sqrt{n} G_{k k}^{\circ}(z)\right\} .
$$

To prove the convergence of $\operatorname{Re} \sqrt{n} G_{k k}^{\circ}(z)$ to the Gaussian random variable $\alpha(z)$ with zero mean and variance $\mu(z)=\operatorname{Var}\{\alpha(z)\}$, it suffices to obtain the asymptotic relation

$$
\frac{d}{d t} F_{n}(t)=-\mu(z) t F_{n}(t)+o(1), n \rightarrow \infty,
$$

uniformly in $t$ on a compact interval.

Differentiating (4.1) with respect to $t$, we obtain

$$
\frac{d}{d t} F_{n}(t)=\frac{i \sqrt{n}}{2}\left(\mathbf{E}\left\{\psi(t) G_{k k}^{\circ}\left(z_{1}\right)\right\}+\mathbf{E}\left\{\psi(t) G_{k k}^{\circ}\left(z_{2}\right)\right\}\right),
$$

where $z_{1}=z, z_{2}=\bar{z}$. To find $\mathbf{E}\left\{\sqrt{n} \psi(t) G_{k k}^{\circ}\left(z_{1}\right)\right\}$, we use Proposition 3.1 with $\Phi=\psi^{\circ}(t) G_{a c}\left(z_{1}\right)$ and repeat the procedure similar to that used in the proof of the preceding theorem. This leads to the relation

$$
\mathbf{E}\left\{\sqrt{n} \psi(t) G_{k k}^{\circ}\left(z_{1}\right)\right\}=\frac{i t F_{n}(t)}{2}\left(\gamma\left(z_{1}, z_{1}\right)+\gamma\left(z_{1}, z_{2}\right)\right)+O\left(n^{-1 / 2}\right),
$$

where the function $\gamma$ is defined in (3.10). This relation and the analogous relation for $\mathbf{E}\left\{\sqrt{n} \psi(t) G_{k k}^{\circ}\left(z_{2}\right)\right\}$ lead to (4.2) with

$$
\mu(z)=4^{-1}\left(S\left(z_{1}, z_{1}\right)+S\left(z_{2}, z_{2}\right)+2 S\left(z_{1}, z_{2}\right)\right) .
$$

Thus, we have proved the theorem for $\operatorname{Re} \sqrt{n} G_{k k}^{\circ}(z)$. Other assertions can be proved in a similar way.

Consider now the arbitrary test function $\varphi: \mathbb{R} \rightarrow \mathbb{R}$. The following analogs of Propositions 1.1 and 1.2 not for the linear statistics $\mathcal{N}_{n}[\varphi]$, but for the elements $\mathcal{N}_{n}[\varphi]_{k k}=\varphi(H)_{k k}$ are valid:

Proposition 4.2. If $\varphi: \mathbb{R} \rightarrow \mathbb{R}, \varphi \in \mathcal{L}$-vector space with the norm $\|\cdot\|$ and we have:

- the variance $V_{n}[\varphi]_{j j}=\operatorname{Var}\left\{\sqrt{n} \mathcal{N}_{n}[\varphi]_{k k}\right\}$ admits uniform in $n$ bound $V_{n}[\varphi]_{k k} \leq$ $C\|\varphi\|^{2}$ for all $\varphi \in \mathcal{L}$

- there exists a dense linear manifold $\mathcal{L}_{1} \subset \mathcal{L}$ such that the Central Limit Theorem is valid for $\sqrt{n} \mathcal{N}_{n}^{\circ}[\varphi]_{k k}$ with $\varphi \in \mathcal{L}_{1}$, i.e., if $Z_{n}[x \varphi]=\mathbf{E}\left\{e^{i x \sqrt{n} \mathcal{N}_{n}^{\circ}[\varphi]_{k k}}\right\}$, 
then there exists a continuous quadratic functional $V_{k k}: \mathcal{L}_{1} \rightarrow \mathbb{R}_{+}$such that uniformly in $x$ on any compact

$$
\lim _{n \rightarrow \infty} Z_{n}[x \varphi]=e^{-x^{2} V[\varphi]_{k k} / 2}, \forall \varphi \in \mathcal{L}_{1},
$$

then $V_{k k}$ admits a continuous extension to $\mathcal{L}$ and the Central Limit Theorem is valid for all $\sqrt{n} \mathcal{N}_{n}^{\circ}[\varphi]_{k k}$ with $\varphi \in \mathcal{L}$.

The proof of this proposition coincides with the proof of Proposition 1.1 in [3] since the statistic $\mathcal{N}_{n}[\varphi]_{k k}$ remains linear in $\varphi$ although it depends not only on the eigenvalues of $H$ but on its eigenvectors too.

Proposition 4.3. For any $s>0$ and $\|\varphi\|_{s}^{2}=\int_{-\infty}^{+\infty}(1+2|k|)^{2 s}|\hat{\varphi}(k)|^{2} d k$, we have $\operatorname{Var}\left\{\mathcal{N}_{n}[\varphi]_{j j}\right\} \leq$

$$
\leq C\|\varphi\|_{s} \int_{0}^{+\infty} d y e^{-y} y^{2 s-1} \int_{-\infty}^{+\infty} \operatorname{Var}\left\{G_{j j}(x+i y)\right\} d x .
$$

The proof of this proposition follows the proof of Proposition 1.2 from [4] with one simple modification: we consider $\operatorname{Var}\left\{\mathcal{N}_{n}[\varphi]_{j j}\right\}$ as a bounded quadratic form for any fixed $n$ generating a positive self-adjoint operator in a proper space. We will present below in all details the proof of the analog of this proposition for the unitary case.

Following [4], we consider the test function $\varphi: \mathbb{R} \rightarrow \mathbb{R}$ from the space $\mathcal{H}_{s}(\mathbb{R})$ possessing the norm

$$
\|\varphi\|_{s}^{2}=\int_{-\infty}^{+\infty}(1+2|p|)^{2 s}|\hat{\varphi}(p)|^{2} d p, s>2 .
$$

Theorem 4.4. Consider the ensembles (1.1) and (1.2) satisfying the condition (2.1) and $\|A \mid\|, B \| \leq M<\infty$, and hence supp $N$ being compact, $\chi_{N}(\lambda)$ being its indicator; the function $\varphi: \mathbb{R} \rightarrow \mathbb{R}, \varphi \in \mathcal{H}_{2+\varepsilon}(\mathbb{R}), \varepsilon>0$ and $\mathcal{N}_{n}[\varphi]_{j j}$ being the corresponding statistics. Then the random variable

$$
\sqrt{n} \mathcal{N}_{n}^{\circ}[\varphi]_{k k}=\sqrt{n} \mathcal{N}_{n}[\varphi]_{k k}-\sqrt{n} \mathbf{E}\left\{\mathcal{N}_{n}[\varphi]_{k k}\right\}
$$

converges in distribution to the Gaussian random variable with zero mean and the variance

$V[\varphi]=\lim _{\eta_{1}, \eta_{2} \downarrow 0} \frac{1}{\pi^{2}} \int_{-\infty}^{+\infty} \int_{-\infty}^{+\infty} \varphi\left(\lambda_{1}\right) \varphi\left(\lambda_{2}\right)\left(\mathbf{I}_{1,2} \cdot S\right)\left(\lambda_{1}+i \eta_{1}, \lambda_{2}+i \eta_{2}\right) \chi_{N}\left(\lambda_{1}\right) \chi_{N}\left(\lambda_{2}\right) d \lambda_{1} d \lambda_{2}$, 
where

$$
\left(\mathbf{I}_{1,2} \cdot S\right)\left(z_{1}, z_{2}\right):=-\operatorname{Re}\left(S\left(z_{1}, z_{2}\right)-S\left(z_{1}, \overline{z_{2}}\right)\right)
$$

and $S\left(z_{1}, z_{2}\right)$ is defined in (2.6) and (2.7) for (1.1) and (1.2), respectively.

P r o o f. First of all, let us note that the bound (3.13) implies

$$
\begin{aligned}
\operatorname{Var}\left\{G_{k k}(x+i y)\right\} & \leq \frac{1}{n} \frac{C}{\left(\operatorname{dist}\left\{x+i y, \operatorname{supp} N_{H_{n}}\right\}\right)^{4}} \\
& \leq \frac{1}{n} \frac{C}{\left(y^{2}+\left(\operatorname{dist}\left\{x, \operatorname{supp} N_{H_{n}}\right\}\right)^{2}\right)^{2}}
\end{aligned}
$$

Thus, due to the fact that supp $N_{H_{n}} \subset[-2 M, 2 M]$ and Proposition 4.3, we have

$$
\begin{aligned}
\operatorname{Var}\left\{\sqrt{n} \mathcal{N}_{n}[\varphi]_{k k}\right\} & \leq C\|\varphi\|_{2+\varepsilon} \int_{0}^{+\infty} d y e^{-y} y^{3+2 \varepsilon} \int_{-\infty}^{+\infty} n \operatorname{Var}\left\{G_{k k}(x+i y)\right\} d x \\
& \leq C\|\varphi\|_{2+\varepsilon} \int_{0}^{+\infty} d y e^{-y} y^{-1+2 \varepsilon} d x \leq C\|\varphi\|_{2+\varepsilon} .
\end{aligned}
$$

Then, according to Proposition 4.2, it suffices to prove the theorem for any dense set in $\mathcal{H}_{2+\varepsilon}([-2 M, 2 M])$, say, for any $\varphi$ analytic in some domain in $\mathbb{C}$ including $[-2 M, 2 M]$, e.g., for a polynomial $\varphi$. Note that due to the Cauchy theorem,

$$
\begin{aligned}
\sqrt{n} \mathcal{N}_{n}^{\circ}[\varphi]_{k k} & =\sqrt{n} \sum_{l=1}^{n}\left(\varphi_{k k}\left(H_{n}\right)-\mathbf{E}\left\{\varphi_{k k}\left(H_{n}\right)\right\}\right) \\
& =\frac{\sqrt{n}}{2 \pi i} \int_{\Gamma} \varphi(z)\left(-G_{k k}(z)+\mathbf{E}\left\{G_{k k}(z)\right\}\right) d z \\
& =-\frac{\sqrt{n}}{2 \pi i} \int_{\Gamma} \varphi(z) G_{k k}^{\circ}(z) d z
\end{aligned}
$$

where $\Gamma$ is any closed contour in the complex plane encircling the segment $[-2 M, 2 M]$ in the real axis. Define the characteristic function

$$
Z_{n}(x)=\mathbf{E}\left\{e_{n}(x)\right\}, x \in \mathbb{R},
$$

where

$$
e_{n}(x)=e^{\left.i x \sqrt{n} \mathcal{N}_{n}^{\circ}[\varphi]\right]_{k k}}=\exp \left\{-\frac{\sqrt{n} x}{2 \pi} \int_{\Gamma} \varphi(z) G_{k k}^{\circ}(z) d z\right\}
$$


Since $Z_{n}(0)=1$ and

$$
e_{n}(x)=1+\int_{0}^{x} e_{n}^{\prime}(y) d y, Z_{n}(x)=1+\int_{0}^{x} Z_{n}^{\prime}(y) d y,
$$

it suffices to prove that there exist the subsequences $\left\{Z_{n_{j}}(x)\right\}$ and $\left\{Z_{n_{j}}^{\prime}(x)\right\}$ that converge uniformly on any finite interval, and

$$
\lim _{n_{j} \rightarrow \infty} Z_{n_{j}}(x)=Z(x), \lim _{n_{j} \rightarrow \infty} Z_{n_{j}}^{\prime}(x)=-x V[\varphi] Z(x) .
$$

Besides, due to the Cauchy theorem,

$$
\begin{aligned}
\frac{d}{d x} e_{n}(x) & =-\frac{\sqrt{n}}{2 \pi} e_{n}(x) \int_{\Gamma} \varphi(z) G_{k k}^{\circ}(z) d z \\
& =-\frac{\sqrt{n}}{2 \pi} \int_{\Gamma_{1}} \varphi\left(z_{1}\right) e_{n}(x) G_{k k}^{\circ}\left(z_{1}\right) d z_{1}, \\
Z_{n}^{\prime}(x) & =-\frac{\sqrt{n}}{2 \pi} \int_{\Gamma_{1}} \varphi\left(z_{1}\right) \mathbf{E}\left\{e_{n}^{\circ}(x) G_{k k}\left(z_{1}\right)\right\} d z_{1},
\end{aligned}
$$

where we choose the contour $\Gamma_{1} \subset D_{M}$ in the domain

$$
D_{M}=\{z \in \mathbb{C}: \rho=\min \operatorname{dist}(z,[-2 M, 2 M])>4 M\} .
$$

To find $\mathbf{E}\left\{e_{n}^{\circ}(x) G_{k k}\left(z_{1}\right)\right\}$ in both cases (1.1) and (1.2), we apply the same procedure as in the previous section and obtain the analog of (3.9) for the case (1.1):

$$
\begin{aligned}
& \operatorname{Cov}\left\{e_{n}^{\circ}(x), G_{k k}\left(z_{1}\right)\right\}=\left\langle e_{n}^{\circ}(x) G_{k k}\left(z_{1}\right)\right\rangle \\
& =-\frac{x Z_{n}(x)}{\sqrt{n} 2 \pi} \int_{\Gamma_{2}} \varphi\left(z_{2}\right) \gamma\left(z_{2}, z_{1}\right) d z_{2}+\sqrt{n} R_{3},
\end{aligned}
$$

where

$$
\begin{aligned}
R_{3}= & \frac{1}{\mathbf{E}\left\{g_{n}\left(z_{1}\right)\right\}}\left(\mathbf{E}\left\{e_{n}^{\circ}(x) g_{n}^{\circ}\left(z_{1}\right) \widetilde{G}_{A}\left(z_{1}\right) U^{*} B U G\left(z_{1}\right)\right\}\right. \\
& \left.-\mathbf{E}\left\{e_{n}^{\circ}(x) \delta_{n, B}^{\circ}\left(z_{1}\right) \widetilde{G}_{A}\left(z_{1}\right) G\left(z_{1}\right)\right\}+\frac{x}{\sqrt{n}} \int_{\Gamma_{2}} \varphi\left(z_{2}\right) R_{4}\left(z_{2}, z_{1}\right) d z_{2}\right)
\end{aligned}
$$

and

$$
\begin{aligned}
R_{4}\left(z_{2}, z_{1}\right) & =\mathbf{E}\left\{e_{n}(x)\left(\widetilde{G}_{A}\left(z_{1}\right) U^{*} B U G\left(z_{2}\right)\right)_{k k}^{\circ}\left(G\left(z_{2}\right) G\left(z_{1}\right)\right)_{k k}^{\circ}\right\} \\
& -\mathbf{E}\left\{e_{n}(x)\left(\widetilde{G}_{A}\left(z_{1}\right) G\left(z_{2}\right)\right)_{k k}^{\circ}\left(G\left(z_{2}\right) U^{*} B U G\left(z_{1}\right)\right)_{k k}^{\circ}\right\}
\end{aligned}
$$




$$
\begin{aligned}
& +\mathbf{E}\left\{e_{n}(x)\left(\widetilde{G}_{A}\left(z_{1}\right) U^{*} B U G\left(z_{2}\right)\right)_{k k}^{\circ}\right\} \mathbf{E}\left\{G\left(z_{2}\right) G\left(z_{1}\right)\right\}_{k k} \\
& -\mathbf{E}\left\{e_{n}(x)\left(\widetilde{G}_{A}\left(z_{1}\right) G\left(z_{2}\right)\right)_{k k}^{\circ}\right\} \mathbf{E}\left\{G\left(z_{2}\right) U^{*} B U G\left(z_{1}\right)\right\}_{k k} \\
& +\mathbf{E}\left\{\widetilde{G}_{A}\left(z_{1}\right) U^{*} B U G\left(z_{2}\right)\right\}_{k k} \mathbf{E}\left\{e_{n}(x)\left(G\left(z_{2}\right) G\left(z_{1}\right)\right)_{k k}^{\circ}\right\} \\
& -\mathbf{E}\left\{\widetilde{G}_{A}\left(z_{1}\right) G\left(z_{2}\right)\right\}_{k k} \mathbf{E}\left\{e_{n}(x)\left(G\left(z_{2}\right) U^{*} B U G\left(z_{1}\right)\right)_{k k}^{\circ}\right\},
\end{aligned}
$$

where the contour $\Gamma_{2} \subset D_{M}$. Besides, for $z \in D_{M}$, we have the bounds

$$
\begin{aligned}
\left|\delta_{A_{n}, B_{n}}(z)\right| & \leq \frac{1}{4},\left|g_{n}(z)\right| \geq \frac{1}{2|z|}, \min \operatorname{dist}\left(h_{A_{n}, B_{n}}(z),[-M, M]\right) \geq 2 M, \\
\left\|G_{n}(z)\right\| & \leq \frac{1}{4 M},\left\|G_{A_{n}, B_{n}}\left(h_{B_{n}, A_{n}}(z)\right)\right\| \leq \frac{1}{2 M},\left|e_{n}(x)\right| \leq 1 .
\end{aligned}
$$

Moreover, by using (3.12)-(3.15), it can be shown that

$$
R_{4}=O\left(n^{-1 / 2}\right)
$$

uniformly for $z_{1,2} \in \Omega, \Omega$-compact, $\Omega \subset D_{M}$. Thus, we obtain

$$
R_{3}=O\left(n^{-1 / 2}\right)
$$

uniformly in $x$ on any finite interval and in $z_{1,2} \in \Omega, \Omega$-compact, $\Omega \subset D_{M}$. Then, using (4.5), we obtain

$$
\begin{aligned}
\sqrt{n} \mathbf{E}\left\{e_{n}^{\circ}(x) G_{k k}\left(z_{1}\right)\right\} & =\frac{x Z_{n}(x)}{2 \pi} \int_{\Gamma_{2}} \varphi\left(z_{2}\right) \gamma\left(z_{2}, z_{1}\right) d z_{2}+O\left(n^{-1 / 2}\right) \\
& =\frac{x Z_{n}(x)}{2 \pi} \int_{\Gamma_{2}} \varphi\left(z_{2}\right) S\left(z_{1}, z_{2}\right) d z_{2}+O\left(n^{-1 / 2}\right) .
\end{aligned}
$$

uniformly in $x$ on any finite interval. Substituting this into (4.4), we obtain

$$
Z_{n}^{\prime}(x)=-\frac{x Z_{n}(x)}{4 \pi} \int_{\Gamma_{1}} \int_{\Gamma_{2}} \varphi\left(z_{1}\right) \varphi\left(z_{2}\right) S\left(z_{1}, z_{2}\right) d z_{1} d z_{2}+O\left(n^{-1 / 2}\right)
$$

uniformly in $x$ on any finite interval in view of the finiteness of the contours $\Gamma_{1,2}$, which completes the proof due to the analyticity of $\varphi\left(z_{1}\right) \varphi\left(z_{2}\right) S\left(z_{1}, z_{2}\right)$ in $z_{1,2}$ for $z_{1,2} \in \mathbb{C} \backslash[-2 M, 2 M]$.

The case (1.2) can be treated in a similar way. 


\section{Covariance of Resolvents of (1.3) and (1.4)}

Now we prove the analogous results for the product. Define $N_{n, S}$ and $N_{n, T}$, the NCMs of $S$ and $T$ for any interval $\Omega \subset \mathbb{T}=\{z \in \mathbb{C}:|z|=1\}$,

$$
\begin{aligned}
& N_{n, S}(\Omega)=\#\left\{s_{l}^{(n)} \in \Omega, l=1, \ldots, n\right\} n^{-1}, \\
& N_{n, T}(\Omega)=\#\left\{t_{l}^{(n)} \in \Omega, l=1, \ldots, n\right\} n^{-1} .
\end{aligned}
$$

We assume that the measures converge weakly to the probability measures $N_{S}$ and $N_{T}$ :

$$
\lim _{n \rightarrow \infty} N_{n, S}=N_{S}, \lim _{n \rightarrow \infty} N_{n, T}=N_{T} .
$$

As in the additive case, there is the way to express the NCM of the product in terms of NCMs of the factors, using, for example, the following result [6]:

Theorem 5.1. If $S$ and $T$ satisfy the condition (5.2), then the $N C M N_{n}$ of (1.3) converges weakly with probability 1 to the non-random probability measure $N$, and the Stieltjes transform

$$
f(z)=\int_{\mathbb{T}} \frac{N(d \mu)}{\mu-z}
$$

of $N$ is a unique solution of the system

$$
\left\{\begin{array}{ccc}
\Delta_{T}(z) & = & f_{S}\left(\frac{z \Delta_{S}(z)}{1+z f(z)}\right), \\
\Delta_{S}(z) & = & f_{T}\left(\frac{z \Delta_{T}(z)}{1+z f(z)}\right), \\
f(z)(1+z f(z)) & = & \Delta_{S}(z) \Delta_{T}(z),
\end{array}\right.
$$

where $f(z), \Delta_{S}(z), \Delta_{T}(z)$ are analytic for the $|z|<1$ functions verifying the conditions

$$
\Delta_{S}(0)=\overline{m_{T}^{(1)}}, \Delta_{T}(0)=\overline{m_{S}^{(1)}} .
$$

Despite its bulkiness, when compared with (2.2) in the additive case, the system (5.4) gives a useful observation:

$\mathrm{R}$ e $\mathrm{m}$ a $\mathrm{rk}$ 5.2. (Divisors of the uniform distribution on $\mathbb{T}$.) If we consider (5.4) as the definition of the free multiplicative convolution $N_{S} \otimes N_{T}=N$ of the measures on $\mathbb{T}$, we can use it to obtain all divisors of uniform distribution on $\mathbb{T}$ with respect to $\otimes$. Indeed, for the $N$-uniform distribution, we have $f(z) \equiv 0$ and

$$
\left\{\begin{array}{ccc}
\Delta_{T}(z) & = & f_{S}\left(z \Delta_{S}(z)\right) \\
\Delta_{S}(z) & = & f_{T}\left(z \Delta_{T}(z)\right), \\
\Delta_{S}(z) \Delta_{T}(z) & = & 0 .
\end{array}\right.
$$


Thus, where are only two possibilities: we have the purely trivial solution $\left(f(z) \equiv 0, \Delta_{S}(z) \equiv 0, \Delta_{T}(z) \equiv 0\right)$, which implies $m_{S}^{(1)}=m_{T}^{(1)}=0$, and at least one of the measures (say, $N_{S}$ ) is uniform. In the last case, $0 \equiv \Delta_{T}(z)=f_{S}(z)=$ $m_{S}^{(1)}, \Delta_{S}(z)=f_{T}(0) \equiv \overline{m_{T}^{(1)}}(\neq 0$ in general) and where are no more options.

Thus we have to distinguish the cases $f(z) \equiv 0$ and $f(z) \neq 0$.

Theorem 5.3. If $S$ and $T$ are non-random satisfying the condition (5.2) and $f(z) \equiv 0$, then we have for $G_{k k}$ and $\left|z_{1,2}\right|<1$,

$$
\operatorname{Cov}\left\{G_{k k}\left(z_{1}\right), G_{k k}\left(z_{2}\right)\right\}=n^{-1} S_{n}\left(z_{1}, z_{2}\right)+r_{n}\left(z_{1}, z_{2}\right),
$$

where

- in the case of (1.3) and $0 \equiv \Delta_{S, T}(z)$,

$$
S_{n}\left(z_{1}, z_{2}\right)=\frac{f_{T}^{\prime}(0)}{1-z_{1} z_{2} f_{S}^{\prime}(0) f_{T}^{\prime}(0)}\left(S_{k k}^{*}\right)^{2},
$$

- $f o r 0 \equiv \Delta_{T}(z)=f_{S}(z), \Delta_{S}(z)=\overline{m_{T}^{(1)}}$,

$$
\begin{aligned}
S_{n}\left(z_{1}, z_{2}\right) & =\left(S G_{S}\left(\overline{m_{T}^{(1)}} z_{1}\right) G_{S}\left(\overline{m_{T}^{(1)}} z_{2}\right)\right)_{k k} \\
& \times\left(f_{T}^{\prime}(0) S_{k k}^{*}-\frac{\left(\overline{m_{T}^{(1)}}\right)^{2}}{\delta z}\left(G_{S}\left(\overline{m_{T}^{(1)}} z_{1}\right)-G_{S}\left(\overline{m_{T}^{(1)}} z_{2}\right)\right)_{k k}\right) ;
\end{aligned}
$$

- in the case of (1.3) and $0 \equiv \Delta_{S}(z)=f_{T}(z)$ and in the case of (1.4),

$$
S_{n}\left(z_{1}, z_{2}\right)=0
$$

and

$$
\left|r_{n}\left(z_{1}, z_{2}\right)\right| \leq C / n^{-3 / 2} .
$$

P r o o f. We use the scheme of the proof of Theorem 2.2: first we consider the ensemble (1.3) and then the ensemble (1.4). In Proposition 3.1, taking $\Phi=$ $\left(\operatorname{Tr} E^{(k, k)} G\left(z_{1}\right)\right)^{\circ} G_{a c}\left(z_{2}\right)$ and using the resolvent identity for the pair $(H, A)$, we obtain

$$
\begin{aligned}
& \left\langle\left(\operatorname{Tr} E^{(k, k)} G\left(z_{1}\right)\right)^{\circ}\left(G\left(z_{2}\right) S\left[X, U^{*} T U\right] G\left(z_{2}\right)\right)_{a c}\right\rangle \\
+ & \left\langle\left(\operatorname{Tr} E^{(k, k)} G\left(z_{1}\right) S\left[X, U^{*} T U\right] G\left(z_{1}\right)\right) G_{a c}\left(z_{2}\right)\right\rangle=0 .
\end{aligned}
$$


Then, taking $X=E^{(a, b)}$ and applying the operation $n^{-1} \sum_{a=1}^{n}$ to the result, we obtain the matrix equality

$$
\begin{gathered}
\left\langle G_{k k}^{\circ}\left(z_{1}\right)\left(1+z_{2} g_{n}\left(z_{2}\right)\right) G\left(z_{2}\right)\right\rangle=\left\langle G_{k k}^{\circ}\left(z_{1}\right) \delta_{n, S}\left(z_{2}\right) U^{*} T U G\left(z_{2}\right)\right\rangle \\
+n^{-1}\left\langle\left[U^{*} T U, G\left(z_{1}\right) E^{(k, k)} G\left(z_{1}\right) S\right] G\left(z_{2}\right)\right\rangle
\end{gathered}
$$

where

$$
\delta_{n, S}=n^{-1} \operatorname{Tr} S G .
$$

Rewrite $G$ by using the resolvent identity for the pair $(W, I)$ as $G=z^{-1}\left(S U^{*} T U G\right.$ $-I)$. After regrouping the terms, we have

$$
\begin{aligned}
& \frac{1+z_{2} f_{n}\left(z_{2}\right)}{z_{2}}\left(S-\rho_{n, S}\left(z_{2}\right) I\right)\left\langle G_{k k}^{\circ}\left(z_{1}\right) U^{*} T U G\left(z_{2}\right)\right\rangle \\
= & n^{-1}\left\langle\left[U^{*} T U, G\left(z_{1}\right) E^{(k, k)} G\left(z_{1}\right) S\right] G\left(z_{2}\right)\right\rangle \\
& +\left\langle G_{k k}^{\circ}\left(z_{1}\right) \delta_{n, B}^{\circ}\left(z_{2}\right) U^{*} T U G\left(z_{2}\right)\right\rangle \\
& -z_{2}\left\langle G_{k k}^{\circ}\left(z_{1}\right) g_{n}^{\circ}\left(z_{2}\right) G\left(z_{2}\right)\right\rangle,
\end{aligned}
$$

where

$$
f_{n}(z)=\mathbf{E}\left\{g_{n}(z)\right\}, \rho_{n, S}(z)=\frac{z \mathbf{E}\left\{\delta_{n, S}(z)\right\}}{1+z f_{n}(z)} .
$$

Besides, we have the bounds

$$
\begin{aligned}
|| G(z) \| & \leq \frac{1}{|1-| z||},\left|g_{n}(z)\right| \leq \frac{1}{|1-| z||},\left|\delta_{n, S}(z)\right| \leq \frac{1}{|1-| z||},|z| \neq 1 \\
\left|\rho_{n, B}(z)\right| & \leq \frac{|z|}{1-2|z|},|z|<\frac{1}{2},\left|\rho_{n, B}(z)\right| \leq \frac{1}{2},|z|<\frac{1}{4}
\end{aligned}
$$

Thus the matrix $S-\rho_{n, S}\left(z_{2}\right) I$ in (5.10) has the inverse

$$
\widetilde{G}_{S}(z)=\left(S-\rho_{n, S}(z) I\right)^{-1}=G_{S}\left(\rho_{n, S}(z)\right)
$$

uniformly in $n$ bounded $\left\|\widetilde{G}_{S}(z)\right\| \leq 2$ for $|z|<1 / 4$. Next, multiplying (5.10) by $\widetilde{G}_{S}\left(z_{2}\right)$ from the left, we obtain the relation

$$
\begin{aligned}
& \left\langle G_{k k}^{\circ}\left(z_{1}\right) U^{*} T U G\left(z_{2}\right)\right\rangle \\
= & \frac{1}{n} \frac{z_{2}}{1+z_{2} f_{n}\left(z_{2}\right)}\left\langle\widetilde{G}_{S}\left(z_{2}\right)\left[U^{*} T U, G\left(z_{1}\right) E^{(k, k)} G\left(z_{1}\right) S\right] G\left(z_{2}\right)\right\rangle \\
& +\left\langle G_{k k}^{\circ}\left(z_{1}\right) \delta_{n, B}^{\circ}\left(z_{2}\right) \widetilde{G}_{S}\left(z_{2}\right) U^{*} T U G\left(z_{2}\right)\right\rangle \\
& -z_{2}\left\langle G_{k k}^{\circ}\left(z_{1}\right) g_{n}^{\circ}\left(z_{2}\right) \widetilde{G}_{S}\left(z_{2}\right) G\left(z_{2}\right)\right\rangle .
\end{aligned}
$$


Multiplying this relation by $\frac{1}{z_{2}} S$ from the left, regrouping the terms according to the resolvent identity and applying then the operation $\operatorname{Tr} E^{(k, k)}$., we obtain

$$
\operatorname{Cov}\left\{G_{k k}\left(z_{1}\right), G_{k k}\left(z_{2}\right)\right\}=\left\langle G_{k k}^{\circ}\left(z_{1}\right) G_{k k}\left(z_{2}\right)\right\rangle=\frac{1}{n} \gamma\left(z_{1}, z_{2}\right)+R_{5},
$$

where

$$
\begin{aligned}
\gamma\left(z_{1}, z_{2}\right)= & \frac{1}{1+z_{2} f_{n}\left(z_{2}\right)}\left(\left\langle S \widetilde{G}_{S}\left(z_{2}\right) U^{*} T U G\left(z_{1}\right)\right\rangle_{k k}\left\langle G\left(z_{1}\right) S G\left(z_{2}\right)\right\rangle_{k k}\right. \\
& \left.-\left\langle S \widetilde{G}_{S}\left(z_{2}\right) G\left(z_{1}\right)\right\rangle_{k k}\left\langle G\left(z_{1}\right) S U^{*} T U G\left(z_{2}\right)\right\rangle_{k k}\right), \\
R_{5}= & \frac{1}{1+z_{2} f_{n}\left(z_{2}\right)}\left(\mathbf{E}\left\{G_{k k}^{\circ}\left(z_{1}\right) \delta_{n, B}^{\circ}\left(z_{2}\right) S \widetilde{G}_{S}\left(z_{2}\right) U^{*} B U G\left(z_{2}\right)\right\}\right. \\
& -z_{2} \mathbf{E}\left\{G_{k k}^{\circ}\left(z_{1}\right) g_{n}^{\circ}\left(z_{2}\right) \widetilde{G}_{A}\left(z_{2}\right) G\left(z_{2}\right)\right\} \\
& +n^{-1} \mathbf{C o v}\left\{\left(S \widetilde{G}_{S}\left(z_{2}\right) U^{*} T U G\left(z_{1}\right)\right)_{k k},\left(G\left(z_{1}\right) S G\left(z_{2}\right)\right)_{k k}\right\} \\
& \left.-n^{-1} \mathbf{C o v}\left\{\left(S \widetilde{G}_{S}\left(z_{2}\right) G\left(z_{1}\right)\right)_{k k},\left(G\left(z_{1}\right) S U^{*} T U G\left(z_{2}\right)\right)_{k k}\right\}\right) .
\end{aligned}
$$

Now the Schwarz inequality for the expectation $\mathbf{E}\{\ldots\},(5.12)$ and the bound $\left\|\widetilde{G}_{S}(z)\right\| \leq 2$ for $|z| \leq 1 / 4$ yield for $|z|,\left|z_{1,2}\right| \leq 1 / 4$,

$$
\begin{aligned}
& \left|R_{5}\right| \leq \frac{16}{3} \operatorname{Var}^{1 / 2}\left\{G_{k k}(z)\right\}\left(\operatorname{Var}^{1 / 2}\left\{g_{n}(z)\right\}+\operatorname{Var}^{1 / 2}\left\{\delta_{n, B}(z)\right\}\right) \\
& +\frac{1}{n} \frac{3}{2}\left(\operatorname{Var}^{1 / 2}\left\{\left(S \widetilde{G}_{S}\left(z_{2}\right) U^{*} T U G\left(z_{1}\right)\right)_{k k}\right\} \operatorname{Var}^{1 / 2}\left\{\left(G\left(z_{1}\right) S G\left(z_{2}\right)\right)_{k k}\right\}\right. \\
& \left.+\operatorname{Var}^{1 / 2}\left\{\left(S \widetilde{G}_{S}\left(z_{2}\right) G\left(z_{1}\right)\right)_{k k}\right\} \operatorname{Var}^{1 / 2}\left\{\left(G\left(z_{1}\right) S U^{*} T U G\left(z_{2}\right)\right)_{k k}\right\}\right) \cdot(5 .
\end{aligned}
$$

Besides, by using Proposition 3.2, we have

$$
\begin{aligned}
\operatorname{Var}\left\{G_{k k}(z)\right\} & \leq \frac{1}{n} \sum_{j, t=1}^{n} \mathbf{E}\left\{\left|\operatorname{Tr} E^{(k, k)} G S U^{*}\left(E^{(t, j)} T-T E^{(j, t)}\right) U G\right|^{2}\right\} \\
& =\frac{1}{n} \sum_{j, t=1}^{n} \mathbf{E}\left\{\left|\left(T U G E^{(k, k)} G S U^{*}\right)_{j t}-\left(U G E^{(k, k)} G S U^{*} T\right)_{t j}\right|^{2}\right\} \\
& \leq \frac{4}{n|1-| z||^{4}},|z| \neq 1
\end{aligned}
$$

and, analogously,

$$
\operatorname{Var}\left\{\left(G\left(z_{1}\right) S G\left(z_{2}\right)\right)_{k k}\right\} \leq \frac{8}{n|1-| z||^{4}},|z| \neq 1,
$$




$$
\begin{aligned}
\operatorname{Var}\left\{\left(G\left(z_{1}\right) S U^{*} T U G\left(z_{2}\right)\right)_{k k}\right\} & \leq \frac{8}{n|1-| z||^{4}},|z| \neq 1, \\
\operatorname{Var}\left\{\left(S \widetilde{G}_{S}\left(z_{2}\right) U^{*} T U G\left(z_{1}\right)\right)_{k k}\right\} & \leq \frac{8}{n|1-| z||^{4}},|z| \leq \frac{1}{4}, \\
\operatorname{Var}\left\{\left(S \widetilde{G}_{S}\left(z_{2}\right) G\left(z_{1}\right)\right)_{k k}\right\} & \leq \frac{8}{\left.n|1-| z\right|^{4}},|z| \leq \frac{1}{4} .
\end{aligned}
$$

On the other hand, by using Proposition 3.2, in the same way, for $|z| \neq 1$ we obtain

$$
\operatorname{Var}\left\{g_{n}(z)\right\}, \operatorname{Var}\left\{\delta_{n, B}(z)\right\}=O\left(n^{-2}\right)
$$

Thus, we have that

$$
R_{5}=O\left(n^{-3 / 2}\right)
$$

uniformly in $|z|,\left|z_{1,2}\right| \leq 1 / 4$. Besides, in [6] it was proved that

$$
\left\langle U^{*} T U G(z)\right\rangle=\widetilde{G}_{S}(z)+O\left(n^{-1}\right)
$$

uniformly in $|z| \leq 1 / 4$. Due to this relation and Proposition 7.1, for $0 \equiv f(z)=$ $\Delta_{S, T}(z)$ we have

$$
\begin{aligned}
\left\langle S \widetilde{G}_{S}\left(z_{2}\right) U^{*} T U G\left(z_{1}\right)\right\rangle_{k k} & =\left(S \widetilde{G}_{S}\left(z_{2}\right) \widetilde{G}_{S}\left(z_{1}\right)\right)_{k k}+O\left(n^{-1}\right) \\
& =\left(S \widetilde{G}_{S}^{2}(0)\right)_{k k}+O\left(n^{-1}\right)=\left(S^{*}\right)_{k k} \\
\left\langle G\left(z_{1}\right) S G\left(z_{2}\right)\right\rangle_{k k} & =\frac{f_{T}^{\prime}(0)}{1-z_{1} z_{2} f_{S}^{\prime}(0) f_{T}^{\prime}(0)} S_{k k}^{*}+O\left(n^{-1}\right) \\
\left\langle S \widetilde{G}_{S}\left(z_{2}\right) G\left(z_{1}\right)\right\rangle_{k k} & =\frac{\rho_{n, S}\left(z_{1}\right)}{z_{1}}\left(S \widetilde{G}_{S}\left(z_{2}\right) \widetilde{G}_{S}\left(z_{1}\right)\right)_{k k}+O\left(n^{-1}\right) \\
& =O\left(n^{-1}\right) .
\end{aligned}
$$

Substituting this into (5.14), we obtain (5.6). Analogously, in the case $0 \equiv f(z)=$ $\Delta_{T}(z)=f_{S}(z), \Delta_{S}(z)=\overline{m_{T}^{(1)}}$, we have

$$
\begin{aligned}
\left\langle S \widetilde{G}_{S}\left(z_{2}\right) U^{*} T U G\left(z_{1}\right)\right\rangle_{k k} & =\left(S G_{S}\left(\overline{m_{T}^{(1)}} z_{2}\right) G_{S}\left(\overline{m_{T}^{(1)}} z_{1}\right)\right)_{k k}+O\left(n^{-1}\right), \\
\left\langle G\left(z_{1}\right) S G\left(z_{2}\right)\right\rangle_{k k} & =f_{T}^{\prime}(0) S_{k k}^{*}+O\left(n^{-1}\right) \\
\left\langle S \widetilde{G}_{S}\left(z_{2}\right) G\left(z_{1}\right)\right\rangle_{k k} & =\overline{m_{T}^{(1)}}\left(S G_{S}\left(\overline{m_{T}^{(1)}} z_{2}\right) G_{S}\left(\overline{m_{T}^{(1)}} z_{1}\right)\right)_{k k}+O\left(n^{-1}\right), \\
\left\langle G\left(z_{1}\right) S U^{*} T U G\left(z_{2}\right)\right\rangle_{k k} & =\frac{\overline{m_{T}^{(1)}}}{\delta z}\left(z_{1} G_{S}\left(\overline{m_{T}^{(1)}} z_{1}\right)-z_{2} G_{S}\left(\overline{m_{T}^{(1)}} z_{2}\right)\right)_{k k}+O\left(n^{-1}\right)
\end{aligned}
$$


and then we obtain (5.7). In the case $0 \equiv f(z)=\Delta_{S}(z)=f_{T}(z)$, we have

$$
\begin{aligned}
\left\langle G\left(z_{1}\right) S G\left(z_{2}\right)\right\rangle_{k k} & =O\left(n^{-1}\right), \\
\left\langle S \widetilde{G}_{S}\left(z_{2}\right) G\left(z_{1}\right)\right\rangle_{k k} & =O\left(n^{-1}\right)
\end{aligned}
$$

and (5.8).

Now we turn to the ensemble (1.4). Following the scheme of the proof of Theorem 2.2 we arrive (cf. (5.10)) to the relation

$$
\begin{aligned}
& \frac{1+z_{2} f_{n}\left(z_{2}\right)}{z_{2}}\left(V^{*} S V-\rho_{n, S}\left(z_{2}\right) I\right)\left\langle G_{k k}^{\circ}\left(z_{1}\right) U^{*} T U G\left(z_{2}\right)\right\rangle \\
& =\frac{1+z_{2} f_{n}\left(z_{2}\right)}{z_{2}}\left\langle G_{k k}^{\circ}\left(z_{1}\right)\right\rangle \\
& +n^{-1}\left\langle\left[U^{*} T U, G\left(z_{1}\right) E^{(k, k)} G\left(z_{1}\right) S\right] G\left(z_{2}\right)\right\rangle \\
& +\left\langle G_{k k}^{\circ}\left(z_{1}\right) \delta_{n, B}^{\circ}\left(z_{2}\right) U^{*} T U G\left(z_{2}\right)\right\rangle-z_{2}\left\langle G_{k k}^{\circ}\left(z_{1}\right) g_{n}^{\circ}\left(z_{2}\right) G\left(z_{2}\right)\right\rangle .
\end{aligned}
$$

Multiplying this relation first by $\widetilde{G}_{V^{*} S V}\left(z_{2}\right)=\left(V^{*} S V-\rho_{n, S}\left(z_{2}\right)\right)^{-1}$ and then by $z_{2}^{-1} V^{*} S V$ from the left, applying then the operation $\operatorname{Tr} E^{(k, k)}$, taking the average $\mathbf{E}\{\ldots\}$ of the result and regrouping the terms, we obtain the relation

$$
\begin{aligned}
& \operatorname{Cov}\left\{G_{k k}\left(z_{1}\right), G_{k k}\left(z_{2}\right)\right\}=\frac{\rho_{n, S}\left(z_{2}\right)}{z_{2}} \mathbf{C o v}\left\{G_{k k}\left(z_{1}\right),\left(\widetilde{G}_{V^{*} S V}\left(z_{2}\right)\right)_{k k}\right\} \\
& +\frac{1}{n} \frac{1}{1+z_{2} f_{n}\left(z_{2}\right)}\left(\mathbf{E}\left\{V^{*} S V \widetilde{G}_{V^{*} S V}\left(z_{2}\right) U^{*} T U G\left(z_{1}\right)\right\}_{k k} \mathbf{E}\left\{G\left(z_{1}\right) V^{*} S V G\left(z_{2}\right)\right\}_{k k}\right. \\
& \left.-\mathbf{E}\left\{V^{*} S V \widetilde{G}_{V^{*} S V}\left(z_{2}\right) G\left(z_{1}\right)\right\}_{k k} \mathbf{E}\left\{G\left(z_{1}\right) V^{*} S V U^{*} T U G\left(z_{2}\right)\right\}_{k k}\right)+R_{6},
\end{aligned}
$$

where $R_{6}=O\left(n^{-3 / 2}\right)$. Using the same arguments as in the proof of Theorem 2.2 , we obtain

$$
\begin{aligned}
& \frac{\rho_{n, S}\left(z_{2}\right)}{z_{2}} \operatorname{Cov}\left\{G_{k k}\left(z_{1}\right),\left(\widetilde{G}_{V^{*} S V}\left(z_{2}\right)\right)_{k k}\right\} \\
& =\frac{\rho_{n, S}\left(z_{1}\right) \rho_{n, S}\left(z_{2}\right)}{z_{1} z_{2}} \operatorname{Cov}\left\{\left(\widetilde{G}_{V^{*} S V}\left(z_{1}\right)\right)_{k k},\left(\widetilde{G}_{V^{*} S V}\left(z_{2}\right)\right)_{k k}\right\}+O\left(n^{-3 / 2}\right) \\
& =\frac{\rho_{n, S}\left(z_{1}\right) \rho_{n, S}\left(z_{2}\right)}{n z_{1} z_{2}}\left(\frac{1}{n} \operatorname{Tr} \widetilde{G}_{S}\left(z_{1}\right) \widetilde{G}_{S}\left(z_{2}\right)-\Delta_{T}\left(z_{1}\right) \Delta_{T}\left(z_{2}\right)\right)+O\left(n^{-3 / 2}\right)=O\left(n^{-3 / 2}\right)
\end{aligned}
$$

for $0 \equiv f(z)$. Moreover, due to (5.18) and Proposition 7.1, we have

$$
\begin{aligned}
\mathbf{E}\left\{G\left(z_{1}\right) V^{*} S V G\left(z_{2}\right)\right\}_{k k} & =O\left(n^{-1}\right), \\
\mathbf{E}\left\{G\left(z_{1}\right) V^{*} S V U^{*} T U G\left(z_{2}\right)\right\}_{k k} & =\frac{\delta(z f)}{\delta z}+O\left(n^{-1}\right)=O\left(n^{-1}\right)
\end{aligned}
$$

for $0 \equiv f(z)$. Thus we finally arrive to $(5.8)$. 
Theorem 5.4. If $S$ and $T$ are non-random, satisfying condition (5.2) and $f(z) \neq 0$, then for $G_{k k}$ and $\left|z_{1,2}\right|<1$ we have

$$
\operatorname{Cov}\left\{G_{k k}\left(z_{1}\right), G_{k k}\left(z_{2}\right)\right\}=n^{-1} S_{n}\left(z_{1}, z_{2}\right)+r_{n}\left(z_{1}, z_{2}\right),
$$

where

- in the case of (1.3),

$$
\begin{aligned}
S_{n}\left(z_{1}, z_{2}\right)= & \frac{\left(\left(\rho_{n, S}\left(z_{1}\right) G_{S}\left(\rho_{n, S}\left(z_{1}\right)\right)-\rho_{n, S}\left(z_{2}\right) G_{S}\left(\rho_{n, S}\left(z_{2}\right)\right)\right)_{k k}\right)^{2}}{\left(z_{1}-z_{2}\right)\left(z_{1} f\left(z_{1}\right)-z_{2} f\left(z_{2}\right)\right)} \\
& \times \frac{\left.z_{2} \rho_{n, S}\left(z_{1}\right)-z_{1} \rho_{n, S}\left(z_{2}\right)\right)}{z_{1} z_{2}\left(\rho_{n, S}\left(z_{1}\right)-\rho_{n, S}\left(z_{2}\right)\right)},
\end{aligned}
$$

- in the case of (1.4), we have (cf. (2.7))

$$
S_{n}\left(z_{1}, z_{2}\right)=\frac{f_{n}\left(z_{1}\right)-f_{n}\left(z_{2}\right)}{z_{1}-z_{2}}-f_{n}\left(z_{1}\right) f_{n}\left(z_{2}\right)
$$

and $\left|r_{n}\left(z_{1}, z_{2}\right)\right| \leq C / n^{-3 / 2}$.

R e m a r k 5.5. (cf. Remark 2.3) Note that in the case $S=\sigma I$, when (1.3) and (1.4) coincide, the expressions (5.21) and (5.22) coincide as well. Therefore we have

$$
\rho_{n, S}(z)=\frac{\sigma z f(z)}{1+z f(z)}, \quad G_{S}\left(\rho_{n, S}(z)\right)=\frac{1}{\sigma-\rho_{n, S}(z)} I=\frac{1+z f(z)}{\sigma} I .
$$

E x a m p l e 5.6. (cf. Example 2.5) Consider the case $S=T$ and the measure $N_{S}(d \lambda)=\alpha \delta(\lambda+1)+(1-\alpha) \delta(\lambda-1), 0 \leq \alpha \leq 1$ for the ensemble (1.4), again having two point masses $\alpha$ and $1-\alpha$ at the points -1 and 1 , respectively. Then (2.2) reduces to the quadratic equation

$$
\left(z^{2} f^{2}(z)+z f(z)\right)\left(z^{-1}+z-2\right)-(2 \alpha-1)^{2}=0
$$

with the solution

$$
z f(z)=-\frac{1}{2}-\frac{\sqrt{\left(z^{-1}+z-2\right)^{2}+4(2 \alpha-1)^{2}\left(z^{-1}+z-2\right)}}{2\left(z^{-1}+z-2\right)}
$$

and the resulting measure

$$
N(d \theta)=|2 \alpha-1| \delta(\lambda-1)+\frac{1}{\pi} \sqrt{\frac{1-2(2 \alpha-1)^{2}-\cos \theta}{1-\cos \theta}} \chi_{\left[\theta_{+}, \theta_{-}\right]}(\theta) d \theta,
$$

where $\chi_{\left[\theta_{+}, \theta_{-}\right]}$is the indicator of the segment $\left[\theta_{-}, \theta_{+}\right]$and

$$
\theta_{+}=\arccos \left(1-2(2 \alpha-1)^{2}\right), \quad \theta_{-}=2 \pi-\arccos \left(1-2(2 \alpha-1)^{2}\right) .
$$


Thus, for this case we have

$$
\begin{aligned}
S\left(z_{1}, z_{2}\right)= & \frac{1}{2 z_{1} z_{2}}+\frac{z_{1}+z_{2}+4(2 \alpha-1)^{2} \frac{z_{1}^{2}+z_{1} z_{2}+z_{2}^{2}-2\left(z_{1}+z_{2}\right)+1}{u\left(z_{1}\right) u\left(z_{2}\right)}}{2 z_{1} z_{2}\left(\frac{w\left(z_{1}\right)}{u\left(z_{1}\right)}+\frac{w\left(z_{2}\right)}{u\left(z_{2}\right)}\right)} \\
& -\frac{1}{4}\left(\frac{1}{z_{1}}+\frac{w\left(z_{1}\right)}{u\left(z_{1}\right)}\right)\left(\frac{1}{z_{2}}+\frac{w\left(z_{2}\right)}{u\left(z_{2}\right)}\right),
\end{aligned}
$$

where $u(z)=(z-1)^{2}, w(z)=\sqrt{\left(z^{-1}+z-2\right)^{2}+4(2 \alpha-1)^{2}\left(z^{-1}+z-2\right)}$.

P r o o f of Theorem 5.4. Following the proof of the previous theorem for the ensemble (1.3) up to the relation (5.13), we use (5.18) and Proposition 7.1 to obtain

$$
\begin{gathered}
\left\langle S \widetilde{G}_{S}\left(z_{2}\right) U^{*} T U G\left(z_{1}\right)\right\rangle_{k k}=\left(S \widetilde{G}_{S}\left(z_{2}\right) \widetilde{G}_{S}\left(z_{1}\right)\right)_{k k}+O\left(n^{-1}\right) \\
=\frac{\left(\rho_{n, S}\left(z_{1}\right) \widetilde{G}_{S}\left(z_{1}\right)-\rho_{n, S}\left(z_{2}\right) \widetilde{G}_{S}\left(z_{2}\right)\right)_{k k}}{\rho_{n, S}\left(z_{1}\right)-\rho_{n, S}\left(z_{2}\right)}+O\left(n^{-1}\right) \\
\left\langle G\left(z_{1}\right) S G\left(z_{2}\right)\right\rangle_{k k}=\frac{\left(\rho_{n, S}\left(z_{1}\right) \widetilde{G}_{S}\left(z_{1}\right)-\rho_{n, S}\left(z_{2}\right) \widetilde{G}_{S}\left(z_{2}\right)\right)_{k k} \delta \Delta_{S}}{\delta z \delta(z f)}+O\left(n^{-1}\right) \\
\left\langle S \widetilde{G}_{S}\left(z_{2}\right) G\left(z_{1}\right)\right\rangle_{k k}=\frac{\rho_{n, S}\left(z_{1}\right)}{z_{1}} \frac{\left(\rho_{n, S}\left(z_{1}\right) \widetilde{G}_{S}\left(z_{1}\right)-\rho_{n, S}\left(z_{2}\right) \widetilde{G}_{S}\left(z_{2}\right)\right)_{k k}}{\rho_{n, S}\left(z_{1}\right)-\rho_{n, S}\left(z_{2}\right)}+O\left(n^{-1}\right) \\
\left\langle G\left(z_{1}\right) S U^{*} T U G\left(z_{2}\right)\right\rangle_{k k}=\frac{\left(z_{1}\left\langle G\left(z_{1}\right)\right\rangle-z_{2}\left\langle G\left(z_{2}\right)\right\rangle\right)_{k k}+O\left(n^{-1}\right)}{\delta z} \\
=\frac{\left(\rho_{n, S}\left(z_{1}\right) \widetilde{G}_{S}\left(z_{1}\right)-\rho_{n, S}\left(z_{2}\right) \widetilde{G}_{S}\left(z_{2}\right)\right)_{k k}}{\delta z}+O\left(n^{-1}\right) .
\end{gathered}
$$

Substituting this into (5.13), we obtain the assertion of the theorem for the ensemble (1.3). To complete the proof for the ensemble (1.4), we follow the path of the proof of the previous theorem up to the relations (3.9)-(5.20) and substitute into (3.9)-(5.20) the relations below:

$$
\begin{aligned}
\mathbf{E}\left\{V^{*} S V \widetilde{G}_{V^{*} S V}\left(z_{2}\right) U^{*} T U G\left(z_{1}\right)\right\}_{k k} & =\frac{\delta(z f)}{\rho_{n, S}\left(z_{1}\right)-\rho_{n, S}\left(z_{2}\right)}+O\left(n^{-1}\right), \\
\mathbf{E}\left\{G\left(z_{1}\right) V^{*} S V G\left(z_{2}\right)\right\}_{k k} & =\frac{\delta \Delta_{S}}{\delta z}+O\left(n^{-1}\right),
\end{aligned}
$$




$$
\begin{aligned}
\mathbf{E}\left\{V^{*} S V \widetilde{G}_{V^{*} S V}\left(z_{2}\right) G\left(z_{1}\right)\right\}_{k k} & =\frac{\rho_{n, S}\left(z_{1}\right)}{z_{1}} \frac{\delta(z f)}{\delta z}+O\left(n^{-1}\right), \\
\mathbf{E}\left\{G\left(z_{1}\right) S V^{*} S V U^{*} T U G\left(z_{2}\right)\right\}_{k k} & =\frac{\delta(z f)}{\delta z}+O\left(n^{-1}\right) .
\end{aligned}
$$

After simple algebra we obtain the assertion of the theorem for the ensemble (1.4).

\section{Central Limit Theorem for the Entries of Functions of (1.3) and (1.4)}

In the same way as in Section 3, we will prove the Central Limit Theorems for the elements of the statistic function $\varphi$ of the matrices (1.3) and (1.4) using the explicit formulas (5.21) and (5.22), respectively. To apply the scheme of the proof of Theorem 4.4 with the dense set of test functions, we have to satisfy the conditions of Proposition 4.2. Thus we need the analog of Proposition 4.3 below to have a priori bound for the variance of the statistic. Let us consider the function $\varphi: \mathbb{T} \rightarrow \mathbb{C}$ from the Hilbert space $\mathcal{H}_{s}(\mathbb{T}), s \geq 0$ with the norm

$$
\|\varphi\|_{s}^{2}=\sum_{k=-\infty}^{+\infty}(1+2|k|)^{2 s}\left|\hat{\varphi}_{k}\right|^{2}, \hat{\varphi}_{k}=\frac{1}{2 \pi} \int_{-\pi}^{\pi} \varphi\left(e^{i \theta}\right) e^{-i k \theta} d \theta .
$$

Proposition 6.1. For any $\varphi \in \mathcal{H}_{s}(\mathbb{T}), s>0$, and any random unitary $W$ we have the bound

$$
\operatorname{Var}\left\{N_{n}[\varphi]_{j j}\right\} \leq C_{s}\|\varphi\|_{s}^{2} \int_{0}^{+\infty} d t e^{-t} t^{2 s-1} \int_{-\pi}^{\pi} \operatorname{Var}\left\{\operatorname{Re} Q_{j j}\left(e^{-t} e^{i \theta}\right)\right\} d \theta
$$

where $Q(z)=(W+z I)(W-z I)^{-1},|z|<1$.

$\mathrm{P}$ r o o f. We just follow the proof of the analogous proposition for the Hermitian case from [4]. Consider the operator $D_{s}: \mathcal{H}_{s}(\mathbb{T}) \rightarrow L^{2}(\mathbb{T})$ defined for any $\psi \in \mathcal{H}_{s}(\mathbb{T})$,

$$
{\widehat{\left(D_{s} \psi\right.}}_{k}=(1+2|k|)^{s} \hat{\psi}_{k}
$$

For fixed $n, \operatorname{Var}\left\{N_{n}[\varphi]_{j j}\right\}$ is a bounded quadratic form in the Hilbert space $\mathcal{H}_{s}(\mathbb{T})$ with the inner product $(u, v)_{s}=\left(D_{s} u, D_{s} v\right)$, where $(\cdot, \cdot)$ denotes the inner product in $L^{2}(\mathbb{T})$. Then there exists a positive self-adjoint operator $\mathcal{V}_{j j}$ which defines the quadratic form

$$
\operatorname{Var}\left\{N_{n}[\varphi]_{j j}\right\}=\left(\mathcal{V}_{j j} \varphi, \varphi\right)=\operatorname{Tr}\left(\Pi_{\varphi} \mathcal{V}_{j j} \Pi_{\varphi}\right),
$$


where $\Pi_{\varphi}$ is the modified projection operator $\Pi_{\varphi} u=\varphi(u, \varphi) /\|\varphi\|_{L^{2}}$. Besides, we have

$$
\operatorname{Tr}\left(\Pi_{\varphi} \mathcal{V}_{j j} \Pi_{\varphi}\right)=\operatorname{Tr}\left(\Pi_{\varphi} D_{s} D_{s}^{-1} \mathcal{V}_{j j} D_{s}^{-1} D_{s} \Pi_{\varphi}\right) \leq\left\|D_{s} \Pi_{\varphi}\right\|^{2} \operatorname{Tr}\left(D_{s}^{-1} \mathcal{V}_{j j} D_{s}^{-1}\right),
$$

where $\left\|D_{s} \Pi_{\varphi}\right\| \leq\left\|D_{s} \varphi\right\|_{L^{2}}=\|\varphi\|_{s}$. On the other hand, for any $u, v \in L^{2}(\mathbb{T})$, we have

$$
\begin{aligned}
\Gamma(2 s)\left(D_{s}^{-2} u, v\right)= & \Gamma(2 s) \sum_{k=-\infty}^{+\infty}(1+2|k|)^{-2 s} \hat{u}_{k} \hat{v}_{k} \\
= & \int_{0}^{+\infty} d t e^{-t} t^{2 s-1} \sum_{k=-\infty}^{+\infty}\left(e^{-t}\right)^{2|k|} \hat{u}_{k} \hat{v}_{k} \\
= & \int_{0}^{+\infty} d t e^{-t} t^{2 s-1}\left(P_{e^{-t}} * u, P_{e^{-t}} * v\right) \\
& =\int_{0}^{+\infty} d t e^{-t} t^{2 s-1} \int_{-\pi}^{\pi} d \theta \int_{-\pi}^{\pi} \int_{-\pi}^{\pi} P_{e^{-t}}(\theta-\lambda) P_{e^{-t}}(\theta-\mu) u(\lambda) \overline{v(\mu)} d \lambda d \mu,
\end{aligned}
$$

where $\Gamma$ denotes the $\Gamma$-function, $\cdot *$ means the convolution of functions, and $P_{r}(\theta)$ is the Poisson kernel with the parameter $0 \leq r<1$,

$$
P_{r}(\theta)=\frac{1-r^{2}}{1-2 r \cos \theta+r^{2}}=\operatorname{Re} \frac{1+r e^{i \theta}}{1-r e^{i \theta}} .
$$

This implies the explicit form of the integral kernel of $\Gamma(2 s)\left(D_{s}^{-2} u, v\right)$,

$$
\Gamma(2 s) D_{s}^{-2}(\lambda, \mu)=\int_{0}^{+\infty} d t e^{-t} t^{2 s-1} \int_{-\pi}^{\pi} d \theta P_{e^{-t}}(\theta-\lambda) P_{e^{-t}}(\theta-\mu)
$$

and

$$
\begin{aligned}
\Gamma(2 s) \operatorname{Tr}\left(D_{s}^{-1} \mathcal{V}_{j j} D_{s}^{-1}\right) & =\int_{0}^{+\infty} d t e^{-t} t^{2 s-1} \int_{-\pi}^{\pi} d \theta\left(\mathcal{V}_{j j} P_{e^{-t}}(\theta-.), P_{e^{-t}}(\theta-.)\right) \\
& =\int_{0}^{+\infty} d t e^{-t} t^{2 s-1} \int_{-\pi}^{\pi} d \theta \operatorname{Var}\left\{N_{n}\left[P_{e^{-t}}(\theta-.)\right]_{j j}\right\} \\
& =\int_{0}^{+\infty} d t e^{-t} t^{2 s-1} \int_{-\pi}^{\pi} \operatorname{Var}\left\{\operatorname{Re} Q_{j j}\left(e^{-t} e^{i \theta}\right)\right\} d \theta,
\end{aligned}
$$

which completes the proof. 
Before formulating the main result of this section, we should note that although the solution of the system (5.4) and the formulas (5.21), (5.22) are defined only for $|z|<1$, in the case $f(z) \neq 0$, we can always define them also for $|z|>1$. Indeed, using the new variables

$$
\begin{aligned}
\psi(z) & =\frac{z f(z)}{1+z f(z)}, \\
\rho_{S, T}(z) & =\frac{z \Delta_{S, T}(z)}{1+z f(z)},
\end{aligned}
$$

one can rewrite the system (5.4) in the form

$$
\left\{\begin{array}{c}
\psi(z)=\psi_{S}\left(\rho_{S}(z)\right) \\
\psi(z)=\psi_{T}\left(\rho_{T}(z)\right) \\
z \psi(z)=\rho_{S}(z) \rho_{T}(z)
\end{array}\right.
$$

where

$$
\psi_{S, T}(z)=\frac{z f_{S, T}(z)}{1+z f_{S, T}(z)} .
$$

On other hand, under the condition $f(z) \neq 0,|z|<1$, using the integral representation (5.3), one can define $\psi(z)$ for $|z|>1$ via the formula

$$
\psi\left(\frac{1}{\bar{z}}\right)=\frac{1}{\overline{\psi(z)}} .
$$

Then, using the system above, we obtain

$$
\rho_{S, T}\left(\frac{1}{\bar{z}}\right)=\frac{1}{\overline{\rho_{S, T}(z)}} .
$$

Thus, in the case $f(z) \neq 0$, the system above and hence the system (5.4) are valid for any $|z| \neq 1$. Moreover, since the corresponding bounds in (5.12) depend in fact on the distance form $z$ to the unit circle, then Theorem 5.4 with the formulas (5.21), (5.22) is also valid for any $\left|z_{1,2}\right| \neq 1$.

The main result of this section is as follows:

Theorem 6.2. If $S$ and $T$ are non-random, satisfying condition (5.2) and $f(z) \neq 0$, and the test function $\varphi: \mathbb{T} \rightarrow \mathbb{R}, \varphi \in \mathcal{H}_{2+\varepsilon}(\mathbb{T}), \varepsilon>0$, then

$$
\sqrt{n} \mathcal{N}_{n}^{\circ}[\varphi]_{k k}=\sqrt{n} \mathcal{N}_{n}[\varphi]_{k k}-\sqrt{n} \mathbf{E}\left\{\mathcal{N}_{n}[\varphi]_{k k}\right\}
$$

converges in distribution to the Gaussian random variable with zero mean and the variance

$$
V[\varphi]=\lim _{r_{1}, r_{2} \uparrow 1} \frac{1}{4 \pi^{2}} \int_{-\pi}^{\pi} \int_{-\pi}^{\pi} \varphi\left(e^{i \theta_{1}}\right) \varphi\left(e^{i \theta_{2}}\right)\left(\mathbf{R}_{1,2} \cdot T\right)\left(r_{1} e^{i \theta_{1}}, r_{2} e^{i \theta_{1}}\right) d \theta_{1} d \theta_{2},
$$


where

$$
\begin{aligned}
T\left(z_{1}, z_{2}\right) & :=z_{1} z_{2} S\left(z_{1}, z_{2}\right) \\
\left(\mathbf{R}_{1,2} \cdot T\right)\left(z_{1}, z_{2}\right) & :=T\left(z_{1}, z_{2}\right)-T\left(z_{1}, \frac{1}{z_{2}}\right)-T\left(\frac{1}{z_{1}}, z_{2}\right)+T\left(\frac{1}{\overline{z_{1}}}, \frac{1}{z_{2}}\right)
\end{aligned}
$$

and $S\left(z_{1}, z_{2}\right)$ is defined in (5.21) and (5.22) for (1.1) and (1.2), respectively.

$\mathrm{P}$ r o o f. As in the proof of Theorem 4.4, we prove the theorem for some dense set in $\mathcal{H}_{2+\varepsilon}(\mathbb{T})$ (say, trigonometric polynomials) and then extend it on the whole $\mathcal{H}_{2+\varepsilon}(\mathbb{T})$ by using Propositions 6.1 and 4.2. The procedure is the same with the difference in the representation of the analytic in $\mathbb{C} \backslash\{0\}$ test function $\varphi$ via the contour integral by the Cauchy formula. We start with the contour $\Gamma=\Gamma_{r} \cup \Gamma_{\beta}^{+} \cup \Gamma_{1 / r} \cup \Gamma_{r}^{-}$(see Fig. 1.(a)) encircling the spectrum of the ensemble (1.3) (or (1.4)) for any realization.

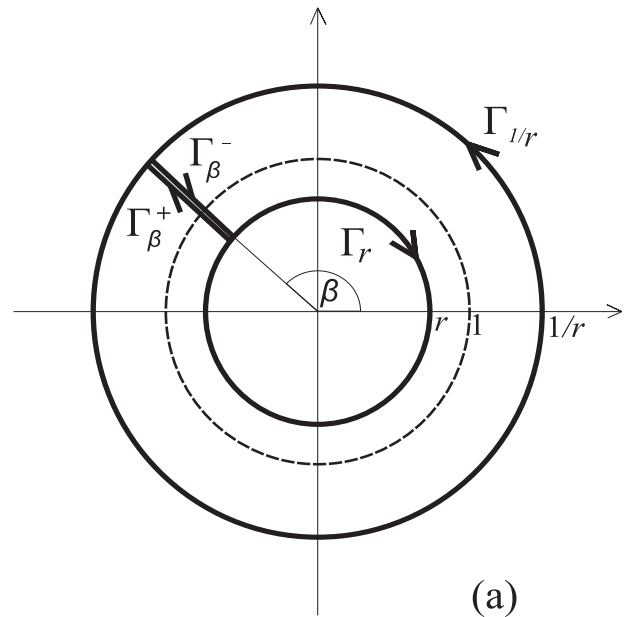

(a)

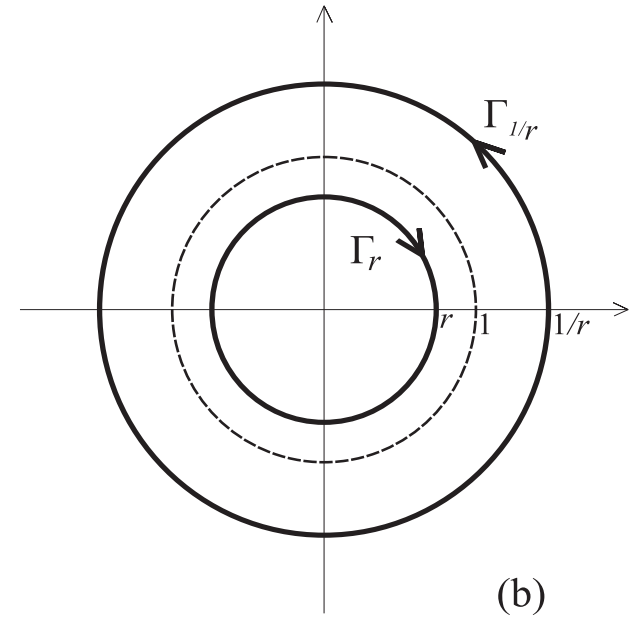

(b)

Fig. 1. Contours (a) and (b)

Of course, this contour has a realization-dependent part (we choose the angle $\beta$ such that the contour $\Gamma$ lies outside the eigenvalues for each realization), but we can cancel the integrals over $\Gamma_{\beta}^{+}$and $\Gamma_{r}^{-}$since they are the same contour integrals in opposite directions. Thus we obtain the integral over the realizationindependent contour $\Gamma_{r} \cup \Gamma_{1 / r}$ (see Fig. 1.(b))

$$
\sqrt{n} \mathcal{N}_{n}^{\circ}[\varphi]_{k k}=-\frac{\sqrt{n}}{2 \pi i} \int_{\Gamma_{r} \cup \Gamma_{1 / r}} \varphi(z) G_{k k}^{\circ}(z) d z
$$




$$
=\frac{\sqrt{n}}{2 \pi} \int_{-\pi}^{\pi}\left(\varphi\left(r e^{i \theta}\right) r e^{i \theta} G_{k k}^{\circ}\left(r e^{i \theta}\right)-\varphi\left(\frac{e^{i \theta}}{r}\right) \frac{e^{i \theta}}{r} G_{k k}^{\circ}\left(\frac{e^{i \theta}}{r}\right)\right) d \theta .
$$

The rest of the proof coincides with the proof of Theorem 4.4.

\section{Appendix}

Proposition 7.1. (i) For the ensemble (1.3) for $|z|<1$ we have in the case $0 \equiv f(z)=\Delta_{S, T}(z)$,

$$
\left\langle G\left(z_{1}\right) S G\left(z_{2}\right)\right\rangle_{k k}=\frac{f_{T}^{\prime}(0)}{1-z_{1} z_{2} f_{S}^{\prime}(0) f_{T}^{\prime}(0)} S_{k k}^{*}+O\left(n^{-1}\right) ;
$$

in the case $0 \equiv f(z)=f_{S}(z)=\Delta_{T}(z)$,

$$
\left\langle G\left(z_{1}\right) S G\left(z_{2}\right)\right\rangle_{k k}=f_{T}^{\prime}(0) S_{k k}^{*}+O\left(n^{-1}\right) ;
$$

in the case $0 \equiv f(z)=f_{T}(z)=\Delta_{S}(z)$,

$$
\left\langle G\left(z_{1}\right) S G\left(z_{2}\right)\right\rangle_{k k}=O\left(n^{-1}\right),
$$

and in the case $f(z) \neq 0$ for $|z|<1$,

$$
\left\langle G\left(z_{1}\right) S G\left(z_{2}\right)\right\rangle_{k k}=\frac{\delta \Delta_{S} \delta \rho_{S}}{\delta z \delta(z f)} S \widetilde{G}_{S}\left(z_{1}\right) \widetilde{G}_{S}\left(z_{2}\right)+O\left(n^{-1}\right) .
$$

(ii) For the ensemble (1.4) we have in the case $0 \equiv f(z)$ for $|z|<1$,

$$
\mathbf{E}\left\{G\left(z_{1}\right) V^{*} S V G\left(z_{2}\right)\right\}_{k k}=O\left(n^{-1}\right),
$$

and in the case $f(z) \neq 0$ for $|z|<1$,

$$
\mathbf{E}\left\{G\left(z_{1}\right) V^{*} S V G\left(z_{2}\right)\right\}_{k k}=\frac{\delta \Delta_{S}}{\delta z}+O\left(n^{-1}\right)
$$

P r o o f. (i) Taking in Proposition 3.1 with $\Phi=\left(G\left(z_{1}\right) S G\left(z_{2}\right)\right)_{a c}$, we obtain

$$
\begin{aligned}
& \left\langle\left(G\left(z_{1}\right) S\left[X, U^{*} T U\right] G\left(z_{1}\right) S G\left(z_{2}\right)\right)_{a c}\right\rangle \\
+ & \left\langle\left(G\left(z_{1}\right) S G\left(z_{2}\right) S\left[X, U^{*} T U\right] G\left(z_{2}\right)\right)_{a c}\right\rangle=0 .
\end{aligned}
$$

Then taking $X=E^{(a, b)}$ and applying the operation $n^{-1} \sum_{a=1}^{n}$ to the result, we get the matrix equality

$$
\begin{aligned}
& \left\langle\delta_{n, S}\left(z_{1}\right) U^{*} T U G\left(z_{1}\right) S G\left(z_{2}\right)\right\rangle-\left\langle\left(1+z_{1} g_{n}\left(z_{1}\right)\right) G\left(z_{1}\right) S G\left(z_{2}\right)\right\rangle \\
+ & \left\langle\left(\frac{1}{n} \operatorname{Tr} G\left(z_{1}\right) S G\left(z_{2}\right) S\right) U^{*} T U G\left(z_{2}\right)\right\rangle \\
- & \left\langle\left(\frac{1}{n} \operatorname{Tr} G\left(z_{1}\right) S G\left(z_{2}\right) S U^{*} T U\right) G\left(z_{2}\right)\right\rangle=0 .
\end{aligned}
$$


Regrouping the terms by using the resolvent identity and centralized values, we obtain

$$
\begin{aligned}
& -\frac{1+z_{1} f_{n}\left(z_{1}\right)}{z_{1}}\left(S-\rho_{n, S}\left(z_{1}\right) I\right)\left\langle U^{*} T U G\left(z_{1}\right) S G\left(z_{2}\right)\right\rangle \\
& +\left\langle\frac{1}{n} \operatorname{Tr} G\left(z_{1}\right) S G\left(z_{2}\right) S\right\rangle\left\langle U^{*} T U G\left(z_{2}\right)\right\rangle \\
& =-\frac{1+z_{1} f_{n}\left(z_{1}\right)}{z_{1}} S\left\langle G\left(z_{2}\right)\right\rangle+\frac{\delta\left(z \Delta_{S}\right)}{\delta z}\left\langle G\left(z_{2}\right)\right\rangle+O\left(n^{-1}\right) .
\end{aligned}
$$

Then taking the inverse $\widetilde{G}_{S}(z)$ and regrouping the terms, we have

$$
\begin{aligned}
& \left\langle U^{*} T U G\left(z_{1}\right) S G\left(z_{2}\right)\right\rangle \\
= & \left\langle G\left(z_{2}\right)\right\rangle+\frac{z_{1}}{1+z_{1} f_{n}\left(z_{1}\right)}\left(\left\langle\frac{1}{n} \operatorname{Tr} G\left(z_{1}\right) S G\left(z_{2}\right) S\right\rangle\right. \\
+ & \left.\frac{\delta \Delta_{S}}{\delta z} \rho_{n, S}\left(z_{2}\right)\right) \widetilde{G}_{S}\left(z_{1}\right) \widetilde{G}_{S}\left(z_{2}\right)+O\left(n^{-1}\right) .
\end{aligned}
$$

After taking the operation $\frac{1}{n} \operatorname{Tr} \cdot$ over (7.6), we obtain the following relation:

$$
\begin{aligned}
& \left\langle\frac{1}{n} \operatorname{Tr} U^{*} T U G\left(z_{1}\right) S G\left(z_{2}\right)\right\rangle \\
- & \frac{z_{1}}{1+z_{1} f_{n}\left(z_{1}\right)} \frac{1}{n} \operatorname{Tr} \widetilde{G}_{S}\left(z_{1}\right) \widetilde{G}_{S}\left(z_{2}\right)\left\langle\frac{1}{n} \operatorname{Tr} G\left(z_{1}\right) S G\left(z_{2}\right) S\right\rangle \\
= & f_{n}(z)-\frac{z_{1} \rho_{n, S}\left(z_{2}\right)}{1+z_{1} f_{n}\left(z_{1}\right)} \frac{\delta \Delta_{S}}{\delta z} \frac{1}{n} \operatorname{Tr} \widetilde{G}_{S}\left(z_{1}\right) \widetilde{G}_{S}\left(z_{2}\right)+O\left(n^{-1}\right) .
\end{aligned}
$$

Note that in the case $f(z) \equiv 0$, we have $\frac{1}{n} \operatorname{Tr} \widetilde{G}_{S}\left(z_{1}\right) \widetilde{G}_{S}\left(z_{2}\right)=\frac{1}{n} \operatorname{Tr} G_{S}^{2}(0)=f_{S}^{\prime}(0)$. On other hand, let us consider the modified resolvent

$$
\hat{G}=U G U^{*}=\left(U S U^{*} T-z I\right)^{-1} .
$$

Due to the trace property, we have the following identities:

$$
\begin{aligned}
\frac{1}{n} \operatorname{Tr} T \hat{G}\left(z_{1}\right) T^{-1} \hat{G}\left(z_{2}\right)= & \frac{1}{z_{1}}\left(\frac{1}{n} \operatorname{Tr} U^{*} T U G\left(z_{1}\right) S G\left(z_{2}\right)-g_{n}\left(z_{2}\right)\right) \\
\left\langle\frac{1}{n} \operatorname{Tr} U S U^{*} \hat{G}\left(z_{1}\right) T^{-1} \hat{G}\left(z_{2}\right)\right\rangle=\frac{1}{z_{1}} & \left(\left\langle\frac{1}{n} \operatorname{Tr} G\left(z_{1}\right) S G\left(z_{2}\right) S\right\rangle-\frac{1}{n} \operatorname{Tr} T^{-1} \widetilde{G}_{T}\left(z_{2}\right)\right) \\
& +O\left(n^{-2}\right) .
\end{aligned}
$$

Then, using the identities above and the left invariant analog of Proposition 3.1 with $\Phi=\left(\hat{G}\left(z_{1}\right) T^{-1} \hat{G}\left(z_{2}\right)\right)_{a c}$ and the procedure similar to that used above, we 
finally obtain

$$
\begin{aligned}
& -\frac{z_{2}}{1+z_{2} f_{n}\left(z_{2}\right)} \frac{1}{n} \operatorname{Tr} \widetilde{G}_{T}\left(z_{1}\right) \widetilde{G}_{T}\left(z_{2}\right)\left\langle\frac{1}{n} \operatorname{Tr} U^{*} T U G\left(z_{1}\right) S G\left(z_{2}\right)\right\rangle \\
& +\left\langle\frac{1}{n} \operatorname{Tr} G\left(z_{1}\right) S G\left(z_{2}\right) S\right\rangle \\
& =\frac{1}{1+z_{2} f_{n}\left(z_{2}\right)}\left(1-\rho_{T}\left(z_{1}\right) z_{2} \frac{\delta \Delta_{S}}{\delta z}\right) \frac{1}{n} \operatorname{Tr} \widetilde{G}_{T}\left(z_{1}\right) \widetilde{G}_{T}\left(z_{2}\right)+O\left(n^{-1}\right) .
\end{aligned}
$$

Thus, for the pair $\left(\left\langle\frac{1}{n} \operatorname{Tr} U^{*} T U G\left(z_{1}\right) S G\left(z_{2}\right)\right\rangle,\left\langle\frac{1}{n} \operatorname{Tr} G\left(z_{1}\right) S G\left(z_{2}\right) S\right\rangle\right)$, we have the following linear system in the case $0 \equiv f(z)=\Delta_{S, T}(z)$ :

$$
\begin{aligned}
\left\langle\frac{1}{n} \operatorname{Tr} U^{*} T U G\left(z_{1}\right) S G\left(z_{2}\right)\right\rangle-z_{1} f_{S}^{\prime}(0)\left\langle\frac{1}{n} \operatorname{Tr} G\left(z_{1}\right) S G\left(z_{2}\right) S\right\rangle & =O\left(n^{-1}\right) \\
-z_{2} f_{T}^{\prime}(0)\left\langle\frac{1}{n} \operatorname{Tr} U^{*} T U G\left(z_{1}\right) S G\left(z_{2}\right)\right\rangle+\left\langle\frac{1}{n} \operatorname{Tr} G\left(z_{1}\right) S G\left(z_{2}\right) S\right\rangle & =f_{T}^{\prime}(0)+O\left(n^{-1}\right) .
\end{aligned}
$$

Solving this system and substituting the solution

$$
\left\langle\frac{1}{n} \operatorname{Tr} G\left(z_{1}\right) S G\left(z_{2}\right) S\right\rangle=\frac{f_{T}^{\prime}(0)}{1-z_{1} z_{2} f_{S}^{\prime}(0) f_{T}^{\prime}(0)}+O\left(n^{-1}\right)
$$

into (7.6), we have for $f(z) \equiv 0$ in all cases

$$
\left\langle U^{*} T U G\left(z_{1}\right) S G\left(z_{2}\right)\right\rangle=\left\langle G\left(z_{2}\right)\right\rangle+z_{1}\left\langle\frac{1}{n} \operatorname{Tr} G\left(z_{1}\right) S G\left(z_{2}\right) S\right\rangle \widetilde{G}_{S}\left(z_{1}\right) \widetilde{G}_{S}\left(z_{2}\right)+O\left(n^{-1}\right),
$$

and hence for $0 \equiv f(z)=\Delta_{S, T}(z)$ we have

$$
\left\langle U^{*} T U G\left(z_{1}\right) S G\left(z_{2}\right)\right\rangle=\left\langle G\left(z_{2}\right)\right\rangle+z_{1} \frac{f_{T}^{\prime}(0)}{1-z_{1} z_{2} f_{S}^{\prime}(0) f_{T}^{\prime}(0)} \widetilde{G}_{S}\left(z_{1}\right) \widetilde{G}_{S}\left(z_{2}\right)+O\left(n^{-1}\right) .
$$

Then, using the consequence of the resolvent identity

$$
\left\langle G\left(z_{1}\right) S G\left(z_{2}\right)\right\rangle=\frac{1}{z_{1}}\left(S\left\langle U^{*} T U G\left(z_{1}\right) S G\left(z_{2}\right)\right\rangle-S\left\langle G\left(z_{2}\right)\right\rangle\right),
$$

we obtain (7.1) for $0 \equiv f(z)=\Delta_{S, T}(z)$.

Analogously, for $0 \equiv f(z)=f_{S}(z)=\Delta_{T}(z)$, we obtain the system

$$
\begin{aligned}
\left\langle\frac{1}{n} \operatorname{Tr} U^{*} \operatorname{TUG}\left(z_{1}\right) S G\left(z_{2}\right)\right\rangle & =O\left(n^{-1}\right), \\
\left\langle\frac{1}{n} \operatorname{Tr} G\left(z_{1}\right) S G\left(z_{2}\right) S\right\rangle & =f_{T}^{\prime}(0)+O\left(n^{-1}\right),
\end{aligned}
$$


and hence we get (7.2). For the case $0 \equiv f(z)=f_{T}(z)=\Delta_{S}(z)$, we obtain the system

$$
\begin{aligned}
\left\langle\frac{1}{n} \operatorname{Tr} U^{*} \operatorname{TUG}\left(z_{1}\right) S G\left(z_{2}\right)\right\rangle & =O\left(n^{-1}\right), \\
\left\langle\frac{1}{n} \operatorname{Tr} G\left(z_{1}\right) S G\left(z_{2}\right) S\right\rangle & =O\left(n^{-1}\right)
\end{aligned}
$$

and then (7.9).

Now we suppose $f(z) \neq 0$ and multiply the relation (7.5) by $S$ and take $\frac{1}{n} \mathrm{Tr}$. over the result. Thus we obtain

$$
\left\langle\frac{1}{n} \operatorname{Tr} G\left(z_{1}\right) S G\left(z_{2}\right) S\right\rangle=\frac{\delta \Delta_{S} \delta\left(z \Delta_{S}\right)}{\delta z \delta(z f)}+O\left(n^{-1}\right) .
$$

Substituting this expression into (7.6) and then using (7.9), we obtain (7.4).

(ii) The first relation in (ii) is obtained from (7.1)-(7.3) by substituting $V^{*} S V$ instead of $S$ and taking $\mathbf{E}\{\cdot\}$ since either $\frac{1}{n} \operatorname{Tr} S^{*}=0$ or $f_{T}^{\prime}(z) \equiv 0$. The second relation in (ii) is obtained from (7.4) in the same way.

Acknowledgement. This work is supported by the Franco-Ukrainian grant Dnipro 2013-2014. The author is thankful to Prof. L. Pastur and Prof. M. Shcherbina for helpful discussions.

\section{References}

[1] L. Pastur and V. Vasilchuk, On the Law of Addition of Random Matrices. - Comm. Math. Phys. 47 (2000), 1-30.

[2] L. Pastur and V. Vasilchuk, On the Law of Addition of Random Matrices: Covariance and the Central Limit Theorem for Traces of Resolvent. - CRM Proc. Lect. Notes 42 (2007), 399-416.

[3] L. Pastur and M. Shcherbina, Eigenvalue Distribution of Random Matrices. Math. Surv. Monogr. 171, RI, AMS, 2011.

[4] M. Shcherbina, Central Limit Theorem for Linear Eigenvalue Statistics of the Wigner and Sample Covariance Random Matrices. - J. Math. Phys., Anal., Geom. 7 (2011), No. 2, 176-192.

[5] G. Chistyakov and F. Götze, The Arithmetic of Distributions in Free Probability Theory. mathOA/0508245.

[6] V. Vasilchuk, On the Law of Multiplication of Random Matrices. - Math. Phys. Anal. Geom. 4 (2001), 1-36. 
[7] A. Lytova and L. Pastur, Fluctuations of Matrix Elements of Regular Functions of Gaussian Random Matrices. — J. Stat. Phys. 134 (2009), 147-159.

[8] A. Lytova, On Non-Gaussian Limiting Laws for Certain Statistics of Wigner Matrices. — J. Math. Phys., Anal., Geom. 9 (2013), No. 4, 536-581.

[9] S. O'Rourke, D. Renfrew, and A. Soshnikov, On Fluctuations of Matrix Entries of Regular Functions of Wigner Matrices with Non-Identicaly Distributed Entries. J. Theor. Probab. 26 (2013), No. 3, 750-780. 